\title{
REDUCED IMPACT LOGGING GUIDELINES FOR INDONESIA
}

Elias

Grahame Applegate

Kuswata Kartawinata

Machfudh

Art Klassen 
Financial support from the International Tropical Timber Organization (ITTO) through the Project PD 12/97 Rev.1 $(F)$, Forest, Science and Sustainability: the Bulungan Model Forest, CIFOR and MacArthur Foundation is gratefully acknowledged.

Cover Photo by Kuswata Kartawinata, Herwasono Soedjito, Plinio Sist and Hari Priyadi.

Art design and production team Roup Purohim and Eko Prianto, Gideon Suharyanto, Widya Prajanthi

() 2001 by Center for International Forestry Research All rights reserved. Published in 2001

Printed by SMK Grafika Desa Putera, Indonesia

\section{ISBN 979-8764-75-7}

\section{Published by}

Center for International Forestry Research Mailing address: P.O. Box 6596 JKPWB, Jakarta 10065, Indonesia Office address: JI. CIFOR, Situ Gede, Sindang Barang, Bogor Barat 16680, Indonesia

Tel.: +62 (251) 622622; Fax: +62 (251) 622100

E-mail: cifor@cgiar.org

Web site: http://mww.cifor.cgiar.org 


\section{FOREWORD}

By

Ir. A. Fattah DS

Director General

Forestry Research and Development Agency (FORDA) Ministry of Forestry

This book, "Reduced Impact Logging Guidelines For Indonesia" has been long awaited by logging practitioners in the field. It is designed as a guide to environmentally sound logging operations in Indonesia. Reduced Impact Logging (RIL), as many forest practitioners perceive, is part of the effort to attain sustainable forest management.

To meet the needs of users, the book uses simple language to make it easy to understand, and is presented in a unique and handy format, making it easy to carry in the field.

It is our pleasure to acknowledge the contribution of the ITTO, through ITTO Project PD 12/97 Rev.1 (F), and to offer our thanks to CIFOR and FORDA for their collaboration in the project's implementation, to PT INHUTANI II for its contribution to research and testing the adoption of RIL techniques, and to the many people who helped at various stages in the writing of this book.

It is our hope that this book will provide a useful reference that will enhance the performance of forest management in Indonesia.

December 2001 


\section{FOREWORD}

\section{By \\ Dr. Mafa Chipeta \\ Deputy Director General of CIFOR}

The world has committed itself to achieving sustainable forest management (SFM), which balances production goals with environmental and social ones. In the field of harvesting, achieving SFM requires environmentally responsible logging, hence the growing interest in reduced impact logging (RIL). The Center for International Forestry Research (CIFOR) is therefore very pleased to have benefited from the financial support of the International Tropical Timber Organization (ITTO) and the strong cooperation of the Forestry Research and Development Organization (FORDA) of the Government of Indonesia to do practical research on RIL in the tropical rain forest of Kalimantan, Indonesia. Unlike common research that is based on small plots, the CIFOR investigations were conducted on a commercial scale on the concessions of PT INHUTANI II, a state-owned timber company.

Central to the successful adoption of the RIL study findings is a clear set of guidelines that define the actions necessary to achieve it. These guidelines, which were adopted by the project, conform to TPTI regulations - the Indonesian silviculture system for dryland natural forest. They also reflect close attention to the ITTO Guidelines 
for the sustainable management of natural tropical forests and the FAO Model Code of forest harvesting practice.

In preparing these Guidelines, CIFOR has been motivated by the real shortage of simple, easy to understand, and practical guidelines on RIL that field operators would find convenient to apply. There is a multitude of regulations and other guidelines on how to harvest forests in a sustainable manner, but few offer the easy format and handy presentation of the present book. In recommending these Guidelines to the industry, CIFOR also invites practitioners to provide feedback on how well their application goes in the field so that continuing improvements can be made. CIFOR commits itself to continue working towards the progressive improvement of RIL practice, in partnership with the Government of Indonesia, industry and others that have an interest in responsible logging.

For making possible the development of these Guidelines as an important achievement of the project, I take this opportunity to express, on behalf of CIFOR, my sincere thanks to the ITTO for funding the project; to PT INHUTANI II for collaborating in the research and in testing the adoption of RIL techniques; and to FORDA for working together with us to make the project a success.

December 2001 


\title{
FOREWORD
}

\author{
By \\ Dr. Untung Iskandar \\ Director General \\ Directorate General of Production Forest Ministry of \\ Forestry
}

Forests, as a national development resource, have a very real function for human life, helping to achieve a dynamic balance of ecological, economic and socio-cultural benefits. For this reason, forests must be managed, protected and utilized in a sustainable manner for the prosperity of all Indonesians, not only current but also future generations.

Logging is one means of utilizing forest located in production forest zones. In practical terms, logging still entails many problems, such as damage to residual stands and the environment from field activities that focus on logging and pay insufficient attention to regeneration and rehabilitation. Environmental damage caused by logging activities includes land opening, soil damage and erosion, residual stand damage and logging waste.

Despite the negative impacts of logging on forests, logging is necessary in order to realize the full economic value of the forest; it also provides a source of funding for forest management. What is needed is a logging method that has a low negative impact. 
One such method is Reduced Impact Logging (RIL). With regard to this, the Directorate General of Production Forest has issued an official memo (No. 274/VI-PHA/2001 dated 23 February 2001) concerning Reduced Impact Logging (RIL). The memo consists of technical guidance for natural forest operation units that intend to implement an environmentally sound logging system.

In anticipation of the successful implementation of RIL, we appreciate the efforts of the ITTO, FORDA CIFOR and INHTANI II in producing these RIL Guidelines.

We hope this book will be of benefit to all.

Jakarta, December 2001 


\section{FOREWORD}

By

Ir. H. Arifin Trihastoyo, MM.

Director General

PT INHUTANI II

The importance of forest values for people is unarguable; forests have a role as a source of support for life. Forests provide multi-benefits - environmental, social and economic. Therefore we have to ensure their sustainability.

Looking at the current situation of severe forest degradation, especially degradation of natural tropical forest, it appears that a common perspective on the forest itself is needed. We should see the forest as a process of natural production, using the sun's energy to process and produce raw materials such as water, minerals, and air in a sustainable manner; these benefits are not a commodity in the form of deposits which can be mined, for example.

We welcome the publication of the book on RIL Guidelines by CIFOR as the result of their collaboration with PT INHUTANI II. We hope that the book will support attempts to maintain sustainability and to enhance the quality of natural forest production processes that will yield optimal and sustainable benefits for human life. 
We also hope that our collaboration can continue, and carry on giving maximal support to the development of forest science and forest management.

Jakarta, November 2001 


\section{FOREWORD}

By

The Authors

The Principles and Practices for Forest Harvesting in Indonesia (2000) have been developed to provide a uniform set of minimum standards for logging practices in the Production and Limited Production Forests in Indonesia. They provide the standard for WHAT is involved in planning and implementing logging activities in natural forest and WHY certain operations should be undertaken. The Reduced Impact Logging (RIL) Guidelines for Indonesia provides the mechanism for HOW the standards will be applied in the field or "how to do the work".

Tractor skidding - mostly by crawlers and skidders - is the most common system (ca. 90\%) used in the Indonesian Selective Cutting and Planting (TPTI) System. Considering the fact that familiarity with more environmentally friendly logging system (such as cable and aerial logging) is still lacking in Indonesia, often due to cost, etc., RIL Guidelines focus on ground-based harvesting which can be implemented in the lowland and hill forests in Indonesia.

This booklet has been prepared for use in the selective cutting silvicultural system of tropical natural forests and based on the Principles and Practices for Forest Harvesting in Indonesia (2000), RIL Pocket Book (Elias, 1999) and Ground Based Reduced-Impact Logging Guidelines for Lowland and Hill Dipterocarp Forest in Indonesia (Sist, 
Dykstra and Fimbel, 1998). Other technical inputs are provided from results of the Reduced-Impact Logging Research of the ITTO funded project on Forest Science and Sustainability: Bulungan Model Forest (PD 12/97 Rev. $1(F)$ ) carried out in Malinau, East Kalimantan, the Code of Practice for Forest Harvesting in Asia-Pacific (1999), Indonesian industry and from actual situations and conditions in the Indonesian forests.

Target groups of the guidelines are production supervisors, RIL planners, block inspectors, road network planners, road construction supervisors, road construction foremen, machine operators, chainsaw operators, tractor operators and their assistants. 


\section{CONTENTS}

FOREWORD

INTRODUCTION

- LOGGING PLANNING 5

- Pre-logging Planning 6

- Forest Area Zoning 8

- Logging Planning 12

- LOGGING OPERATIONS 51

- Supervision of Operations 52

- Felling Operations 57

- Skidding and Landing Operations 73

- MAINTENANCE, CAMP HYGIENEANDSAFETY 89

- Maintenance and Servicing 90

- Camp Higiene 95

- Safety 98

- POST-LOGGING ACTIVITIES 101

- Road Closure 102

- Skidtrail Closure 103

- Temporary Bridge Closure 104

- Quarry Closure 106

- Landing Closure 107

- Camp and Workshop Closure 108

- Road Maintenance 109

CITED REFERENCES 113

$\begin{array}{ll}\text { FURTHERREADING } & 114\end{array}$ 


\section{INTRODUCTION}

\section{Background to the RIL Guidelines for Indonesia}

In Indonesia, forest management and logging operations are regulated under the Indonesian Selective Cutting and Planting System (Sistem Tebang Pilih Tanam Indonesia/ $T P T I)$. RIL practices are recommended under TPTI; however, these are seldom applied in the field for numerous reasons, including :

1. Lack of regulatory control over harvesting practices.

2. Limited specificity in how to conduct RIL techniques.

3. Lack of understanding of the benefits of RIL.

4. Lack of understanding of the steps necessary to implement RIL and lack of specific technical skills.

There is general recognition that existing logging practices need to be improved to ensure a viable residual stand for the successive cutting cycles. As an International Tropical Timber Organization (ITTO) member, such recognition by Indonesian foresters is linked with ITTO's "Year 2000 Objectives" of achieving sustainable forest management.

Progress towards sustainable forest management will be promoted by the implementation of RIL techniques, which aim at minimising soil disturbance, impacts on wildlife, and damage to the residual stand. 


\section{What is RIL?}

RIL is a systematic approach to planning, implementing, monitoring and evaluating logging.

RIL embraces a host of improved road building, felling and skidding practices.

RIL requires forethought and skill, and a policy environment that encourages them.

\section{Why RIL?}

Forest managers are increasingly endeavouring to improve their practices and implement higher standards of forest management. One means of doing this to implement RIL techniques. 


\section{There are a number of reasons why RIL is recommended for implementing RIL, etc.}
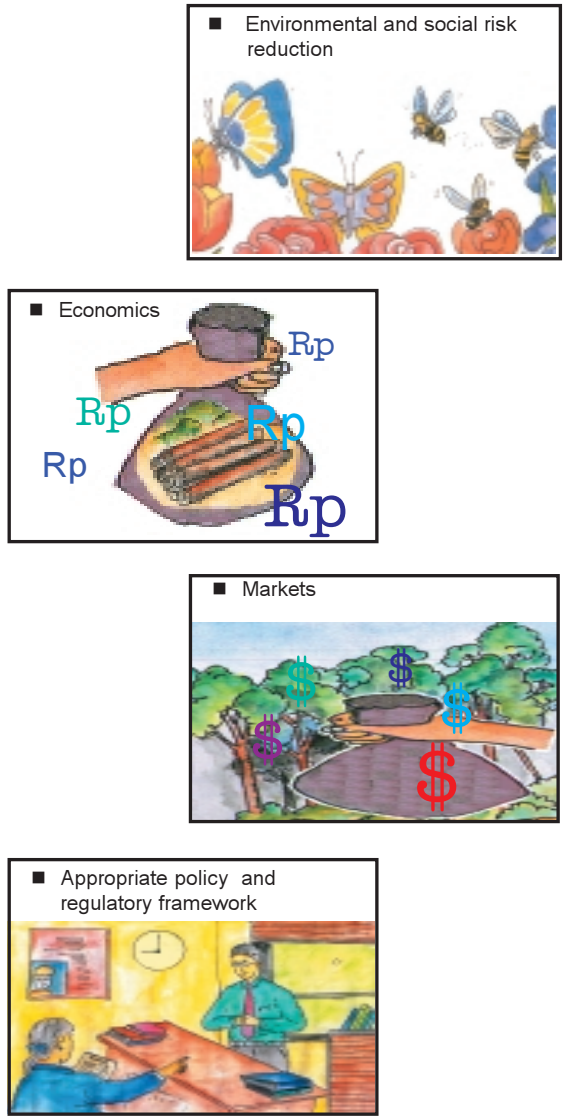

Reduced Impact logging guidelines for indonesia 


\section{RIL IMPLEMENTATION AND TPTI}

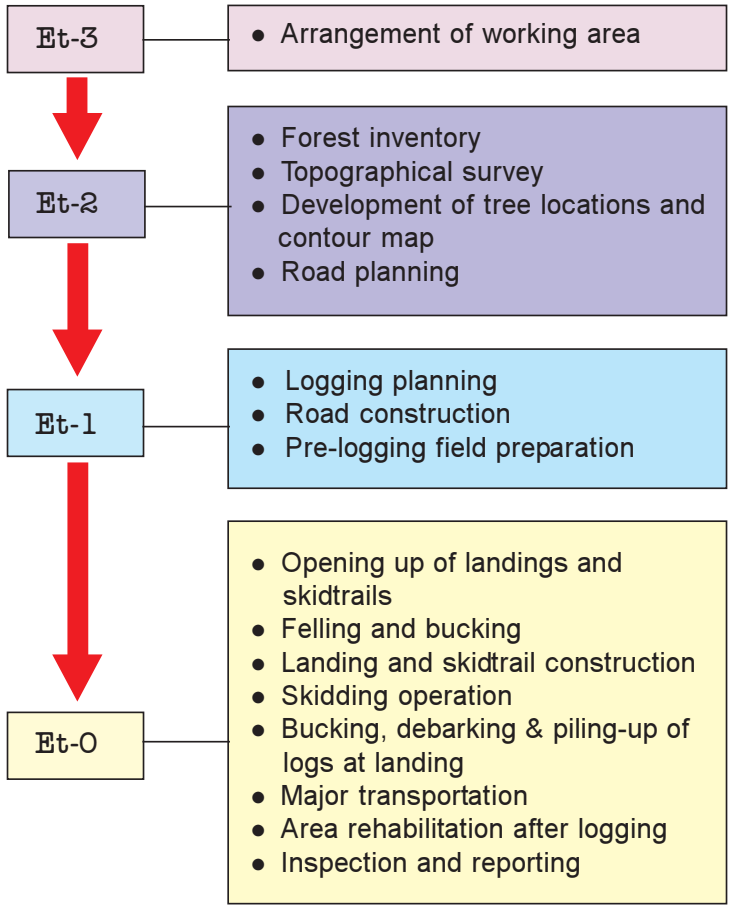

Et-0 : logging time (year); (-) before logging 


\section{LOGGING PLANNING}

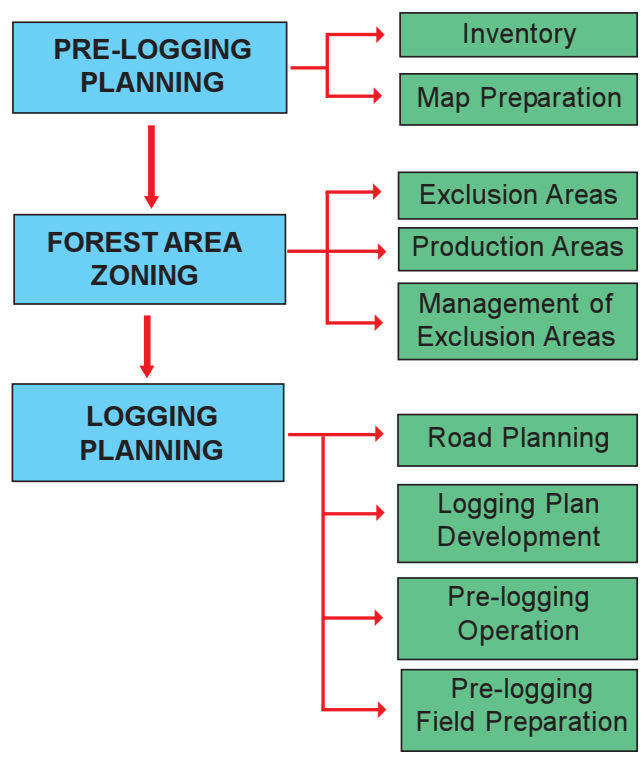




\section{PRE-LOGGING PLANNING}

\section{Inventory}

Undertake a forest inventory, map preparation and stock mapping.

The following manuals provide details of appropriate methods:

- Pedoman dan Petunjuk Teknis Tebang Pilih Tanam Indonesia (TPTI) pada Hutan Alam Daratan

(Departemen Kehutanan RI, 1993)

- Petunjuk Dasar dalam Timber Cruising dan Survei Topografi (Ruslim, 1998)

- Prosedur Survei Topografi Hutan (TFF\&APHI, 2001)

\section{Map Preparation}

Contour and stock mapping at a scale of 1:2 $000-1: 5000$ with contour line intervals of $5-10 \mathrm{~m}$ can be produced manually or by using computer-based software, e.g:

- FIEPLP (Forest Inventory and Product Linking Programme) from TROPENBOS Foundation Project

- GENESIS and GENAMAP Programme from SFMPGTZ Project

- ROADENG Software

- SIPTOP (Sistem Informasi Pohon dan Topografi) from PT. INHUTANI I 
Example of field sheets for data to be recorded as part of the inventory and topographic survey.

Tally sheet of inventory

\begin{tabular}{|c|c|c|c|c|c|c|c|c|}
\hline $\begin{array}{c}\text { Tree } \\
\text { Number }\end{array}$ & $\begin{array}{c}\text { Species } \\
\text { Code }\end{array}$ & $\begin{array}{c}\text { Diam } \\
(\mathrm{cm})\end{array}$ & $\begin{array}{c}\text { Height } \\
(\mathrm{m})\end{array}$ & Code & $\begin{array}{c}\text { Vol } \\
(\mathrm{m} 3)\end{array}$ & $\mathrm{X}$ & $\mathrm{Y}$ & Note \\
\hline & & & & & & & & \\
\hline 5 & $\mathrm{MD}$ & 40 & 18 & 4 & & & & \\
\hline 4 & $\mathrm{MD}$ & 55 & 16 & 1 & & & & \\
\hline 3 & $\mathrm{NYR}$ & 37 & 16 & 4 & & & & \\
\hline 2 & $\mathrm{MD}$ & 25 & 12 & 4 & & & & \\
\hline 001 & $\mathrm{MM}$ & 80 & 22 & 1 & & & & \\
\hline
\end{tabular}

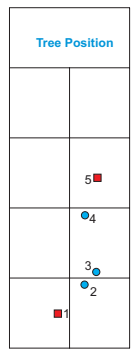

Tally sheet of topo-survey

\begin{tabular}{|c|c|c|c|c|c|c|}
\hline Station & $\begin{array}{c}\text { Azim } \\
\left({ }^{\circ}\right)\end{array}$ & $\begin{array}{l}\text { Horizontal } \\
\text { Distance }\end{array}$ & $\begin{array}{l}\text { Slope } \\
(\%)\end{array}$ & $\begin{array}{c}\text { Heigth } \\
\text { Difference (m) }\end{array}$ & $\begin{array}{l}\text { Elevation } \\
\quad(\mathrm{m})\end{array}$ & $\begin{array}{l}\text { Elev. } \\
\text { Corr. }\end{array}$ \\
\hline \multicolumn{7}{|l|}{3} \\
\hline & 360 & 20.4 & +19 & & & \\
\hline \multicolumn{7}{|l|}{2} \\
\hline & 360 & 20.4 & +19 & & & \\
\hline \multicolumn{7}{|l|}{1} \\
\hline & 360 & 10.1 & +14 & & & \\
\hline 0 & & 10.5 & +33 & & 690 & \\
\hline
\end{tabular}

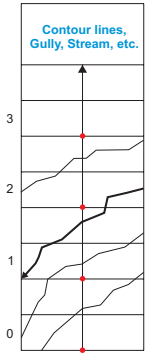




\section{FOREST AREA ZONING}

\section{Exclusion Areas}

- Identification of exclusion areas is important for defining and locating the net productive area for timber production

- Timber production zone = total area of a forest management unit - exclusion areas

- The timber production zone forms the basis for the determination of the Annual Allowable Cut (AAC)

- Exclusion zones, include the following:

- Cultural areas

- Shorelines, lagoons, lakes and water storage areas

- Landslip areas

- Designated watercourses

* Conservation and protection zone

* Community forests and local community zones

- Biological diversity conservation zone

* Wildlife conservation zone

* Scientific research zone

* Buffer zone, e.g: 


\section{Production Areas}

- An example of a forest zoning map

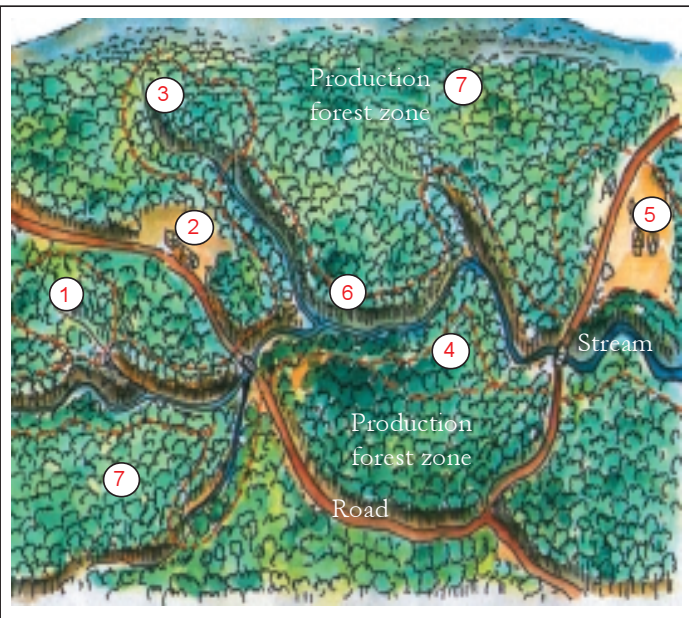

Legend:

1. Biological diversity conservation zone

2,5 . Town surrounded by a community use forest zone

3. Scientific studies forest zone

4. Wildlife conservation zone

6. Watershed conservation zone

7. Production forest zone 
- Buffers for exclusion areas

\begin{tabular}{|l|l|}
\hline & Minimum Width for Buffer Zones \\
\hline Cultural areas & $30 \mathrm{~m}$ \\
\hline $\begin{array}{l}\text { Lakes } \\
\text { Lagoons } \\
\text { Shorelines } \\
\text { Water storage areas }\end{array}$ & $\begin{array}{l}\text { Slope }<17 \%=50 \mathrm{~m} \\
\text { Slope }>17 \%=100 \mathrm{~m}\end{array}$ \\
\hline $\begin{array}{l}\text { Designated } \\
\text { watercourses }\end{array}$ & $\begin{array}{l}\text { Class } 1 \text { stream }=30 \text { m each side } \\
\text { Class 2 stream }=20 \text { m each side } \\
\text { Class 3 stream }=10 \text { m each side }\end{array}$ \\
\hline Waterways & $\begin{array}{l}\text { Catchment area 2 ha, no extraction within } 5 \mathrm{~m} \\
\text { each side }\end{array}$ \\
\hline
\end{tabular}

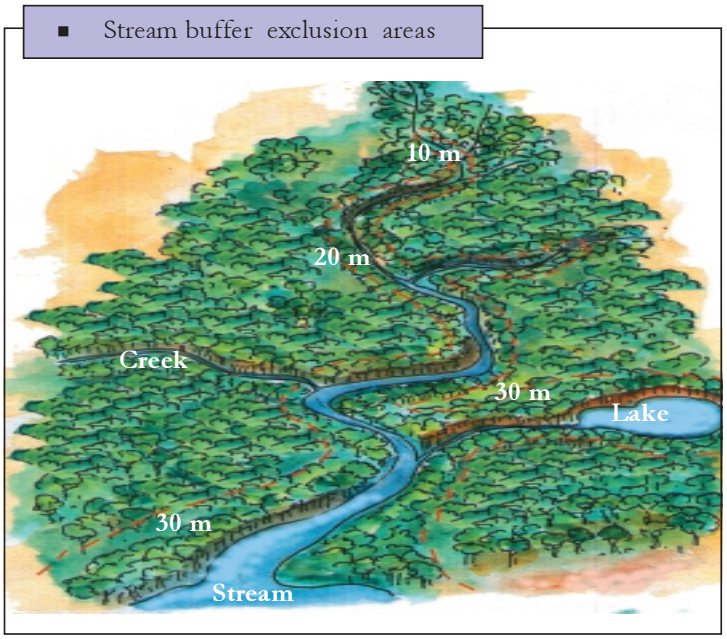




\section{Management of Exclusion Areas}

Areas excluded from logging are managed as follows :

- No trees to be felled within exclusion areas and their buffer zones

- Machine access is prohibited, except where watercourse crossings are permitted

- No earthworks or spoil from earthworks is to fall within the exclusion areas

- No logging debris is to be pushed into the exclusion areas

- Trees should be felled away from buffer zones and watercourses

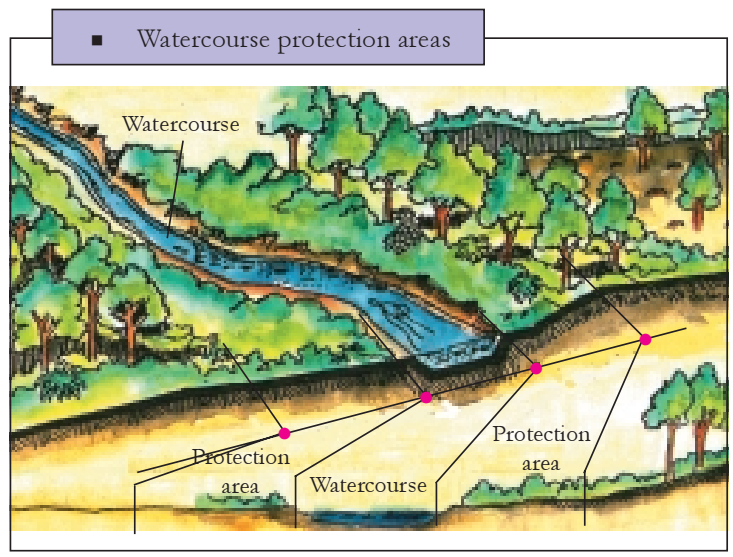




\section{LOGGING PLANNING}

\section{Road Planning}

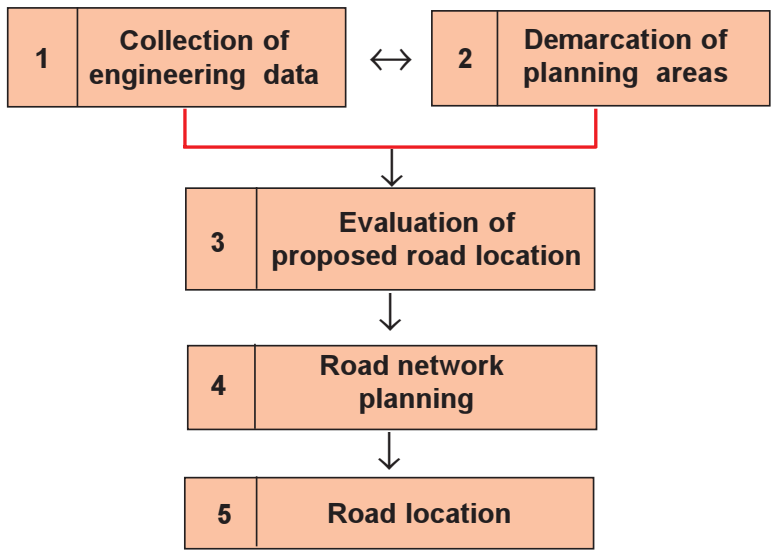

The following references provide details of how to undertake road planning:

- A Manual for the Planning, Design and Construction of Forest Roads in Step Terrain (FAO, undated)

- Pembukaan Wilayah Hutan (Elias, 1997) 


\section{- Collection of engineering data}

Important data to be collected consist of:

- Aerial photographs

- Topographic map at a scale of 1:5000 - 1:25000

- Forest map at a scale 1:25 000 or 1:50 000

- Soil map

- Geological map

- Forest Opening Up Guidelines and Regulations

- Road construction costs

- Timber harvesting costs and timber prices

- Forest Management Plan

- Demarcation of planning area

An example of planning area demarcation

- Existing road

- - $=$ : Planning area boundaries 
- Evaluation of proposed road location

- Need to identify:

- Access points to public roads and existing forest roads

- Proposed road location and logging areas

- Areas with gentle slopes to assist with location, better alignment and log landings

- Stream crossing points

- Gravel deposits (quarries)

- Steep slopes

- Swamps

- Landslides

- Deep canyons

- Protected forests

An example of positive and negative cardinal points/zones

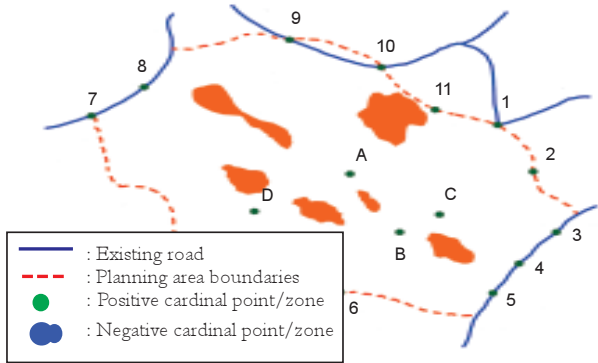




\section{- Road network planning}

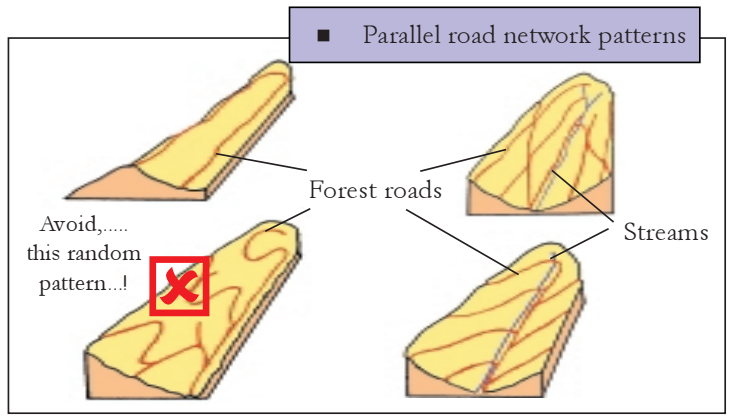

- Circular road network patterns
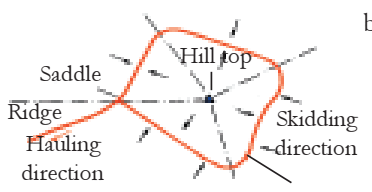

b.

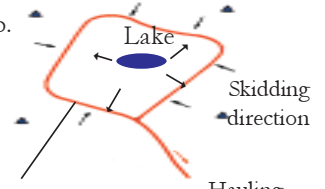

Hauling

direction

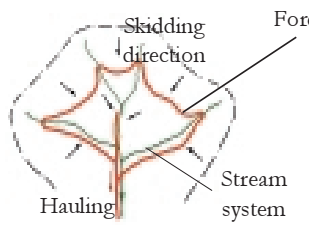

d.

direction

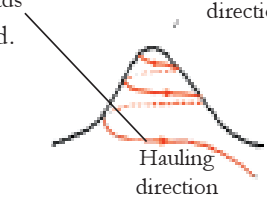



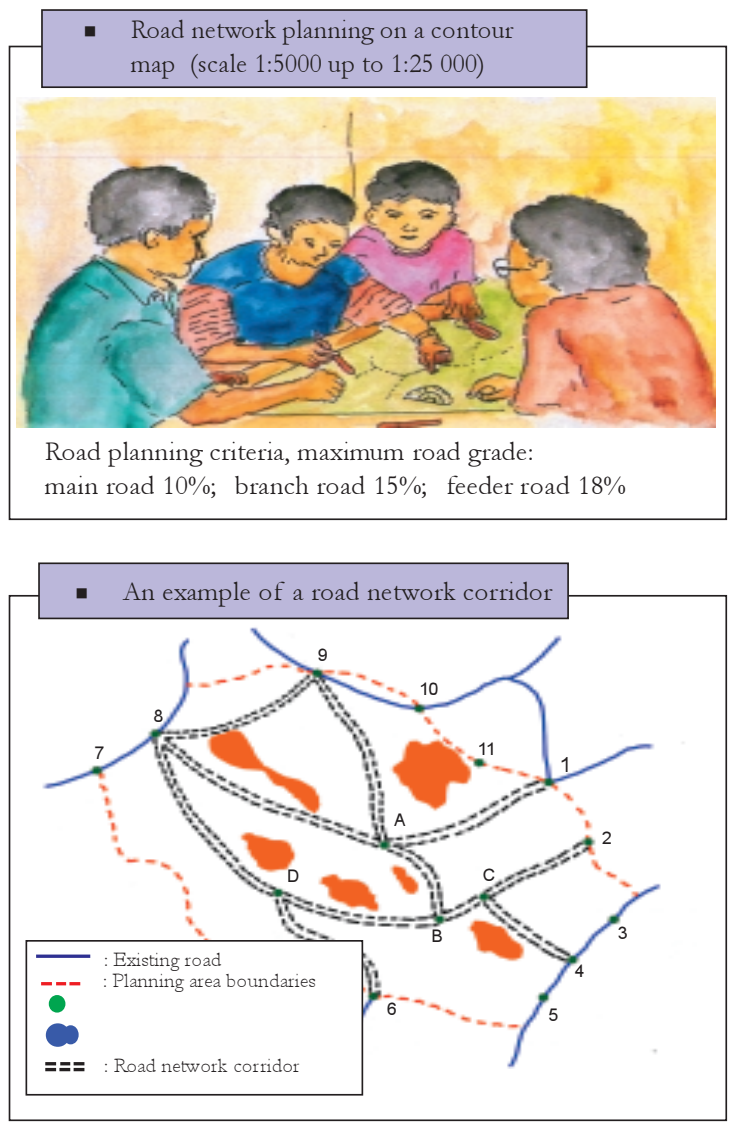
- Choose! road alignment on stable areas.
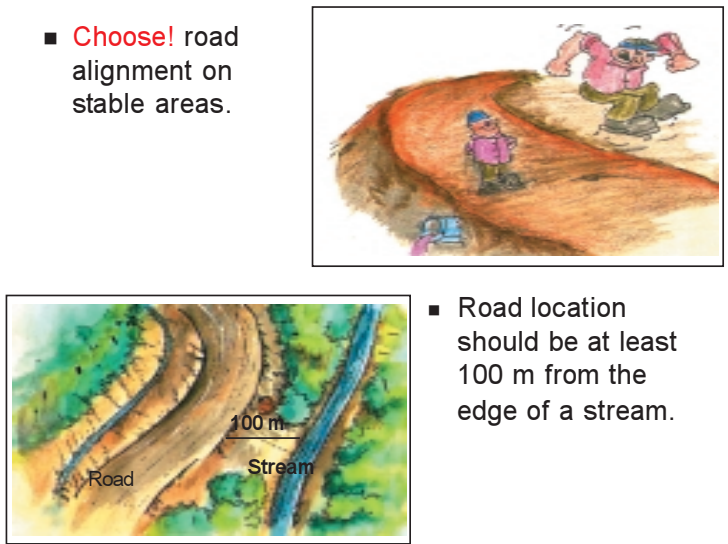

- Road location should be at least $100 \mathrm{~m}$ from the edge of a stream.

- Avoid! areas of cultural importance, steep areas and swamps.

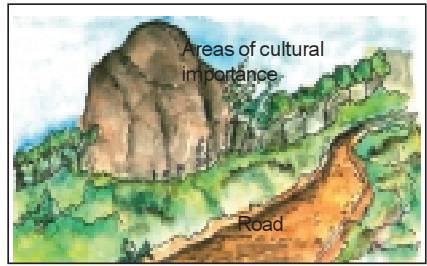

- Avoid! sensitive landslide areas.

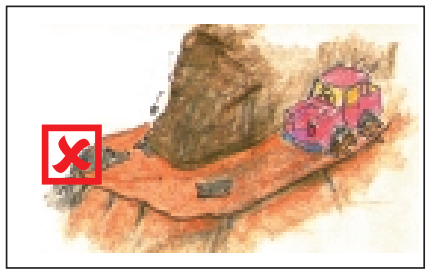




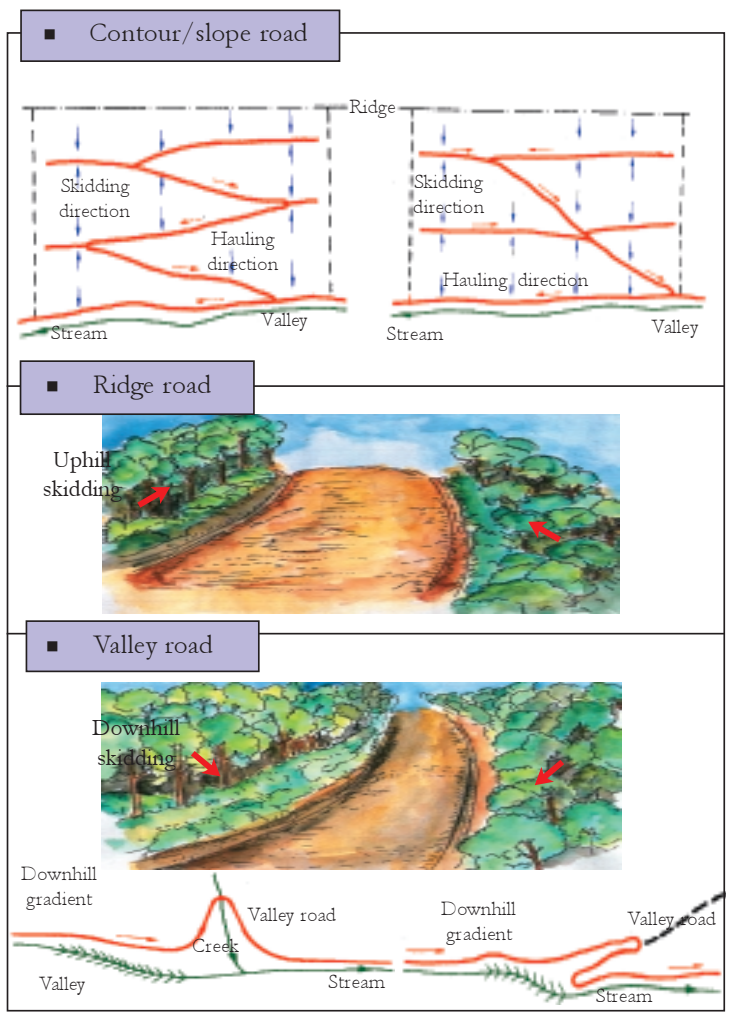


Conversion table for slope measurement

\begin{tabular}{|c|c|c|c|c|c|c|c|c|c|c|c|c|c|}
\hline \multirow{2}{*}{$\begin{array}{l}\text { Deg- } \\
\text { ree } \\
\left({ }^{\circ}\right)\end{array}$} & \multirow{2}{*}{$\begin{array}{l}\text { Per } \\
\text { cent } \\
(\%)\end{array}$} & \multicolumn{12}{|c|}{ Distance (m) } \\
\hline & & 4 & 5 & 6 & 7 & 8 & 9 & 10 & 20 & 30 & 40 & 50 & 100 \\
\hline 1 & 1.7 & 0.00 & 0.00 & 0.00 & 0.00 & 0.00 & 0.00 & 0.00 & 0.00 & 0.00 & 0.01 & 0.01 & 0.02 \\
\hline 2 & 3.5 & 0.00 & 0.00 & 0.00 & 0.00 & 0.00 & 0.01 & 0.01 & 0.01 & 0.02 & 0.02 & 0.03 & 0.06 \\
\hline 3 & 5.2 & 0.01 & 0.01 & 0.01 & 0.01 & 0.01 & 0.01 & 0.01 & 0.03 & 0.04 & 0.05 & 0.07 & 0.14 \\
\hline 4 & 7.0 & 0.01 & 0.01 & 0.01 & 0.02 & 0.02 & 0.02 & 0.02 & 0.05 & 0.07 & 0.10 & 0.12 & 0.24 \\
\hline 5 & 8.7 & 0.02 & 0.02 & 0.02 & 0.03 & 0.03 & 0.03 & 0.04 & 0.08 & 0.11 & 0.15 & 0.19 & 0.38 \\
\hline 6 & 10.5 & 0.02 & 0.03 & 0.03 & 0.04 & 0.04 & 0.05 & 0.05 & 0.11 & 0.16 & 0.22 & 0.27 & 0.55 \\
\hline 7 & 12.3 & 0.03 & 0.04 & 0.04 & 0.05 & 0.06 & 0.07 & 0.07 & 0.15 & 0.22 & 0.30 & 0.37 & 0.75 \\
\hline 8 & 14.1 & 0.04 & 0.05 & 0.06 & 0.07 & 0.08 & 0.09 & 0.10 & 0.19 & 0.29 & 0.39 & 0.49 & 0.97 \\
\hline 9 & 15.8 & 0.05 & 0.06 & 0.07 & 0.09 & 0.10 & 0.11 & 0.12 & 0.25 & 0.37 & 0.49 & 0.62 & 1.23 \\
\hline 10 & 17.6 & 0.06 & 0.08 & 0.09 & 0.11 & 0.12 & 0.14 & 0.15 & 0.30 & 0.46 & 0.61 & 0.76 & 1.52 \\
\hline 11 & 19.4 & 0.07 & 0.09 & 0.11 & 0.13 & 0.15 & 0.17 & 0.18 & 0.37 & 0.55 & 0.73 & 0.92 & 1.84 \\
\hline 12 & 21.3 & 0.09 & 0.11 & 0.13 & 0.15 & 0.17 & 0.20 & 0.22 & 0.44 & 0.66 & 0.87 & 1.09 & 2.19 \\
\hline 13 & 23.1 & 0.10 & 0.13 & 0.15 & 0.18 & 0.21 & 0.23 & 0.26 & 0.51 & 0.77 & 1.03 & 1.28 & 2.56 \\
\hline 14 & 24.9 & 0.12 & 0.15 & 0.18 & 0.21 & 0.24 & 0.27 & 0.30 & 0.59 & 0.89 & 1.19 & 1.49 & 2.97 \\
\hline 15 & 26.8 & 0.14 & 0.17 & 0.20 & 0.24 & 0.27 & 0.31 & 0.34 & 0.68 & 1.02 & 1.36 & 1.70 & 3.41 \\
\hline 16 & 28.7 & 0.15 & 0.19 & 0.23 & 0.27 & 0.31 & 0.35 & 0.39 & 0.77 & 1.16 & 1.55 & 1.94 & 3.87 \\
\hline 17 & 30.6 & 0.17 & 0.22 & 0.26 & 0.31 & 0.35 & 0.39 & 0.44 & 0.87 & 1.31 & 1.75 & 2.18 & 4.37 \\
\hline 18 & 32.5 & 0.20 & 0.24 & 0.29 & 0.34 & 0.39 & 0.44 & 0.49 & 0.98 & 1.47 & 1.96 & 2.45 & 4.89 \\
\hline 19 & 34.4 & 0.22 & 0.27 & 0.33 & 0.38 & 0.44 & 0.49 & 0.54 & 1.09 & 1.63 & 2.18 & 2.72 & 5.45 \\
\hline 20 & 36.4 & 0.24 & 0.30 & 0.36 & 0.42 & 0.48 & 0.54 & 0.60 & 1.21 & 1.81 & 2.41 & 3.02 & 6.03 \\
\hline 21 & 38.4 & 0.27 & 0.33 & 0.40 & 0.46 & 0.53 & 0.60 & 0.66 & 1.33 & 1.99 & 2.66 & 3.32 & 6.64 \\
\hline 22 & 40.4 & 0.29 & 0.36 & 0.44 & 0.51 & 0.58 & 0.66 & 0.73 & 1.46 & 2.18 & 2.91 & 3.64 & 7.28 \\
\hline 23 & 42.4 & 0.32 & 0.40 & 0.48 & 0.56 & 0.64 & 0.72 & 0.79 & 1.59 & 2.38 & 3.18 & 3.97 & 7.95 \\
\hline 24 & 44.5 & 0.35 & 0.43 & 0.52 & 0.61 & 0.69 & 0.78 & 0.86 & 1.73 & 2.59 & 3.46 & 4.32 & 8.65 \\
\hline 25 & 46.6 & 0.37 & 0.47 & 0.56 & 0.66 & 0.75 & 0.84 & 0.94 & 1.87 & 2.81 & 3.75 & 4.68 & 9.37 \\
\hline 26 & 48.8 & 0.40 & 0.51 & 0.61 & 0.71 & 0.81 & 0.91 & 1.01 & 2.02 & 3.04 & 4.05 & 5.06 & 10.12 \\
\hline 27 & 51.0 & 0.44 & 0.54 & 0.65 & 0.76 & 0.87 & 0.98 & 1.09 & 2.18 & 3.27 & 4.36 & 5.45 & 10.90 \\
\hline 28 & 53.2 & 0.47 & 0.59 & 0.70 & 0.82 & 0.94 & 1.05 & 1.17 & 2.34 & 3.51 & 4.68 & 5.85 & 11.71 \\
\hline 29 & 55.4 & 0.50 & 0.63 & 0.75 & 0.88 & 1.00 & 1.13 & 1.25 & 2.51 & 3.76 & 5.02 & 6.27 & 12.54 \\
\hline 30 & 57.7 & 0.54 & 0.67 & 0.80 & 0.94 & 1.07 & 1.21 & 1.34 & 2.68 & 4.02 & 5.36 & 6.70 & 13.40 \\
\hline 31 & 60.1 & 0.57 & 0.71 & 0.86 & 1.00 & 1.1 & 1.29 & 1.43 & 2.86 & 4.28 & 5.71 & 7.14 & 14.28 \\
\hline 32 & 62.5 & 0.61 & 0.76 & 0.91 & 1.06 & 1.22 & 1.37 & 1.52 & 3.04 & 4.56 & 6.08 & 7.60 & 15.20 \\
\hline 33 & 64.9 & 0.65 & 0.81 & 0.97 & 1.13 & 1.29 & 1.45 & 1.61 & 3.23 & 4.84 & 6.45 & 8.07 & 16.13 \\
\hline 34 & 67.5 & 0.68 & 0.85 & 1.03 & 1.20 & 1.37 & 1.54 & 1.71 & 3.42 & 5.13 & 6.84 & 8.55 & 17.10 \\
\hline 35 & 70.0 & 0.72 & 0.90 & 1.09 & 1.27 & 1.45 & 1.63 & 1.81 & 3.62 & 5.43 & 7.23 & 9.04 & 18.08 \\
\hline 36 & 72.7 & 0.76 & 0.95 & 1.15 & 1.34 & 1.53 & 1.72 & 1.91 & 3.82 & 5.73 & 7.64 & 9.55 & 19.10 \\
\hline 37 & 75.4 & 0.81 & 1.01 & 1.21 & 1.41 & 1.61 & 1.81 & 2.01 & 4.03 & 6.04 & 8.05 & 10.07 & 20.14 \\
\hline 38 & 78.1 & 0.85 & 1.06 & 1.27 & 1.48 & 1.70 & 1.91 & 2.12 & 4.24 & 6.36 & 8.48 & 10.60 & 21.20 \\
\hline 39 & 81.0 & 0.89 & 1.11 & 1.34 & 1.56 & 1.78 & 2.01 & 2.23 & 4.46 & 6.69 & 8.91 & 11.14 & 22.29 \\
\hline 40 & 83.9 & 0.94 & 1.17 & 1.40 & 1.64 & 1.87 & 2.11 & 2.34 & 4.68 & 7.02 & 9.36 & 11.70 & 23.40 \\
\hline 41 & 86.9 & 0.98 & 1.23 & 1.47 & 1.72 & 1.96 & 2.21 & 2.45 & 4.91 & 7.36 & 9.81 & 12.26 & 24.53 \\
\hline 42 & 90.0 & 1.03 & 1.28 & 0.54 & 1.80 & 2.05 & 2.31 & 2.57 & 5.14 & 7.71 & 10.27 & 12.84 & 25.69 \\
\hline 43 & 93.3 & 1.07 & 1.34 & 1.61 & 1.88 & 2.15 & 2.42 & 2.69 & 5.37 & 8.06 & 10.75 & 13.43 & 26.86 \\
\hline 44 & 96.6 & 1.12 & 1.40 & 1.68 & 1.96 & 2.25 & 2.53 & 2.81 & 5.61 & 8.42 & 11.23 & 14.03 & 28.07 \\
\hline
\end{tabular}


- Road marking

\begin{tabular}{|l|l|}
\hline \multicolumn{1}{|c|}{ Category } & \multicolumn{1}{c|}{ Feild Marking } \\
\hline Main road & $\begin{array}{l}\text { Centerline with 1 vertical stripe as high as possible on } \\
\text { two sides aligned with the road direction (red colour) }\end{array}$ \\
\hline Branch road and feeder & $\begin{array}{l}\text { Centerline with 1 vertical stripe as high as possible on } \\
\text { two sides aligned with the road direction (red colour) }\end{array}$ \\
\hline Log landing & $\begin{array}{l}\text { Marked with L and number as per harvesting plan on tree } \\
\text { at location (red colour) }\end{array}$ \\
\hline Stream crossing (bridges) & Marked on trees with B (red colour) \\
\hline Stream crossing (culverts) & Marked on trees with C (red colour) \\
\hline
\end{tabular}

- Marking of zero line on the ground

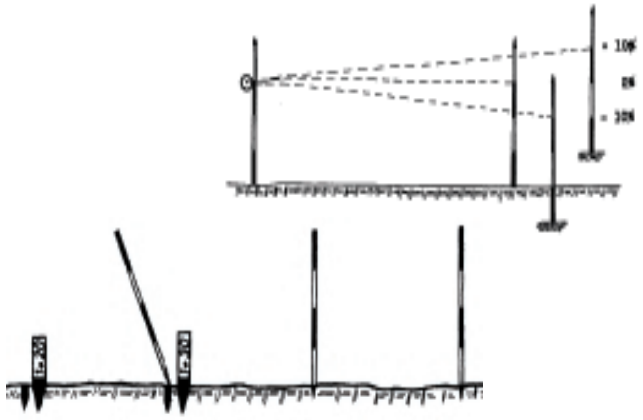

The zero line is determined using a clinometer and is marked on the ground with stakes 

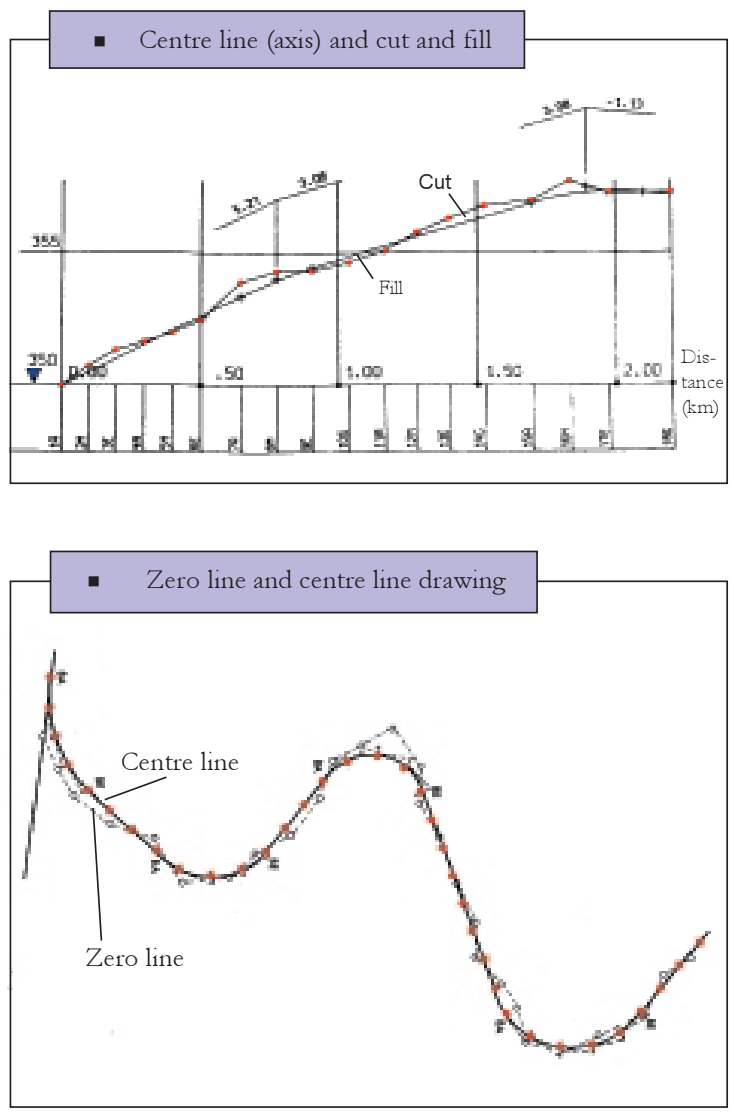


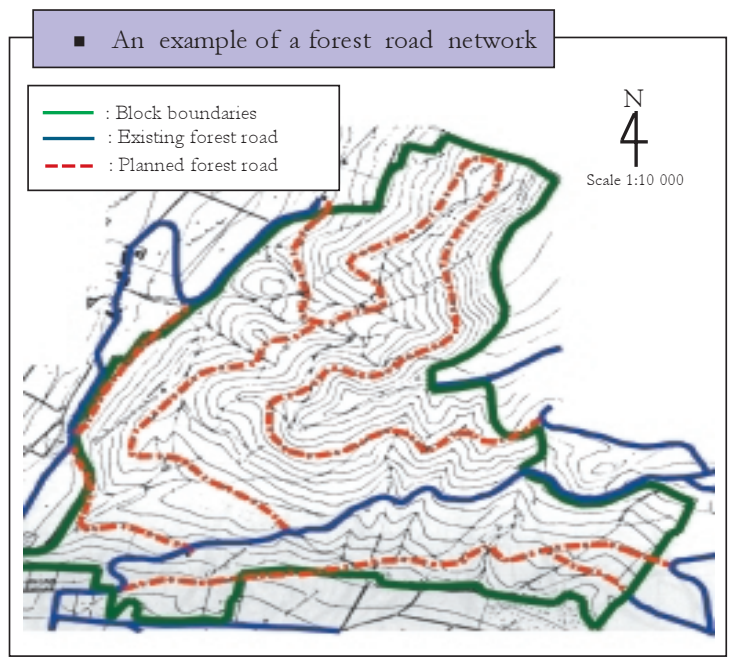




\section{Logging Plan Development}

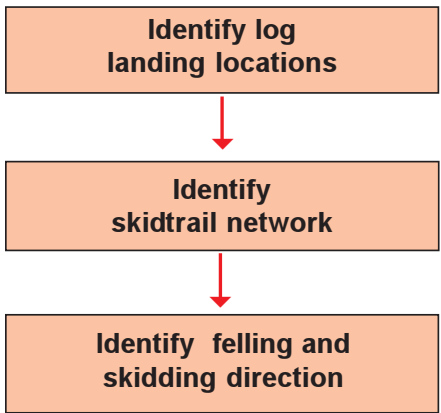




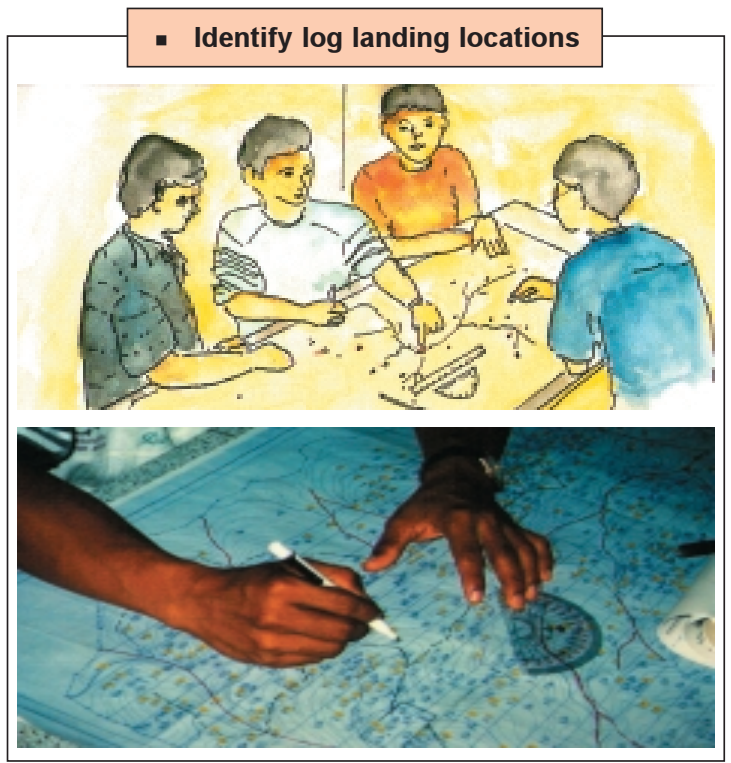

Preferred landings are:

1. Large, flat area with slope of up to $6^{\circ}$

2. Top of hills

3. Not located in Protected Areas

4. Not near streams or watercourses 


\section{Identify skidtrail network}

1. Skidtrails are designed as straight as possible following the contours.

2. Skidtrails should avoid steep areas, ravines, swamps and unstable ground.

3. Skidtrails should minimise the number of stream crossings. If a skidtrail crosses a stream, a small bridge should be constructed.

4. Skidtrails are designed to be used as intensively as possible.

5. Slopes of skidtrails must not exceed $45 \%$.

6. Skidtrail network must avoid exclusion areas.

7. Skidding distances should be minimised.

8. Maximum width of skidtrails is $4 \mathrm{~m}$. 

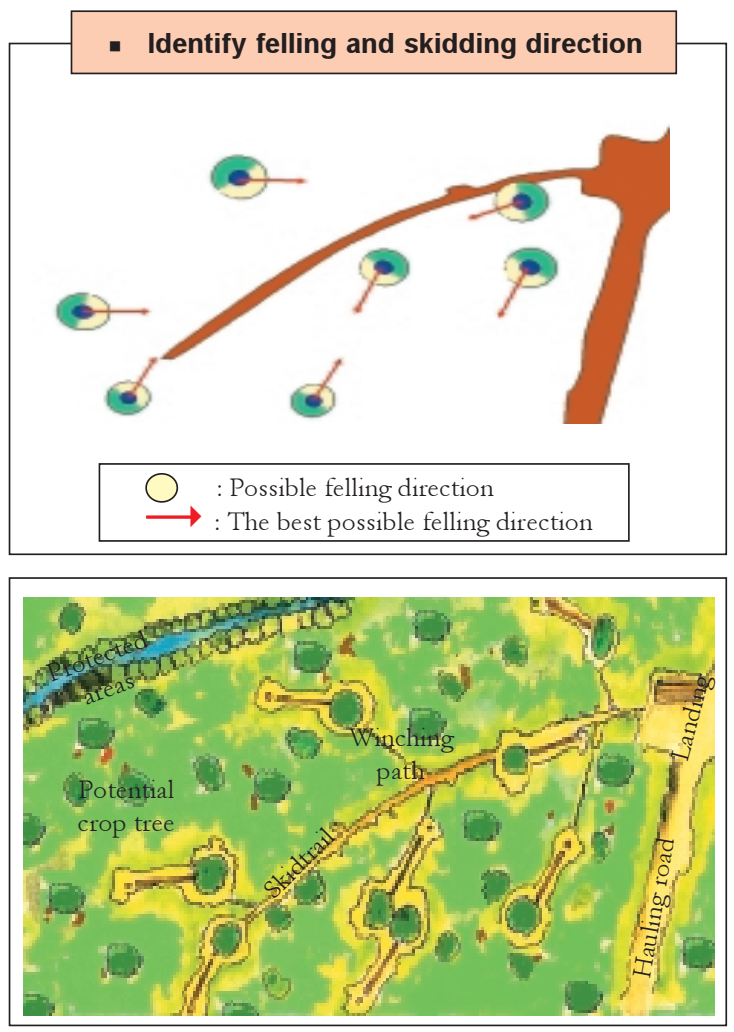


\section{An example a Logging Plan}

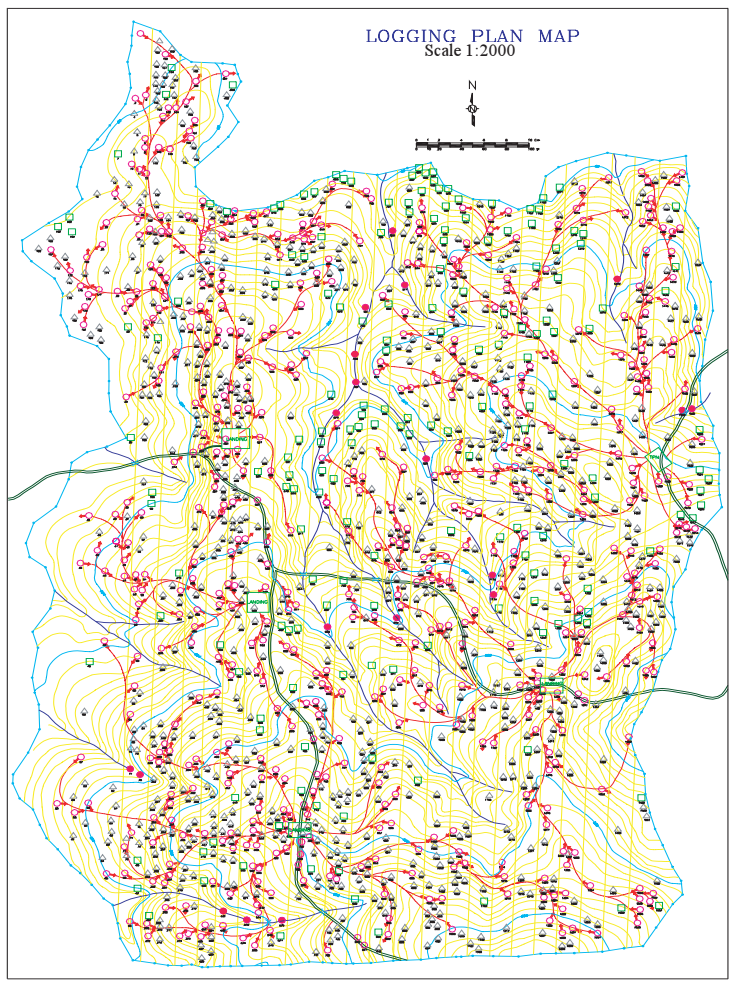

\begin{tabular}{|c|c|c|c|c|c|c|c|c|c|}
\hline$\square$ & COUPAPTVENT BOUHWWES & $\square$ & STREAYS & $\square$ & pFootected tress & 347 & TREES NUMEEA & 1 & FFIIND \\
\hline & CRRISING UNES & $\mathrm{D}_{\mathrm{S}}$ & mucrous tretes & 0 & 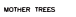 & $\square$ & SKOTRAC & 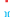 & grapje \\
\hline and & CONIDLS uns & $\square$ & HAVVFSTAR:FS TAEZS & $\square$ & 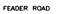 & $\square$ & LANOINO & & \\
\hline
\end{tabular}




\section{Pre-Logging Operation}

Road specifications

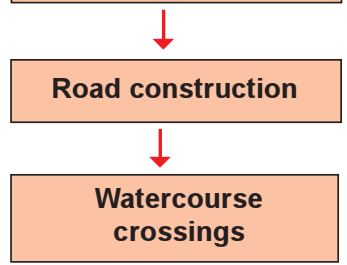




\section{- Road specifications}

- Cross section of contour road

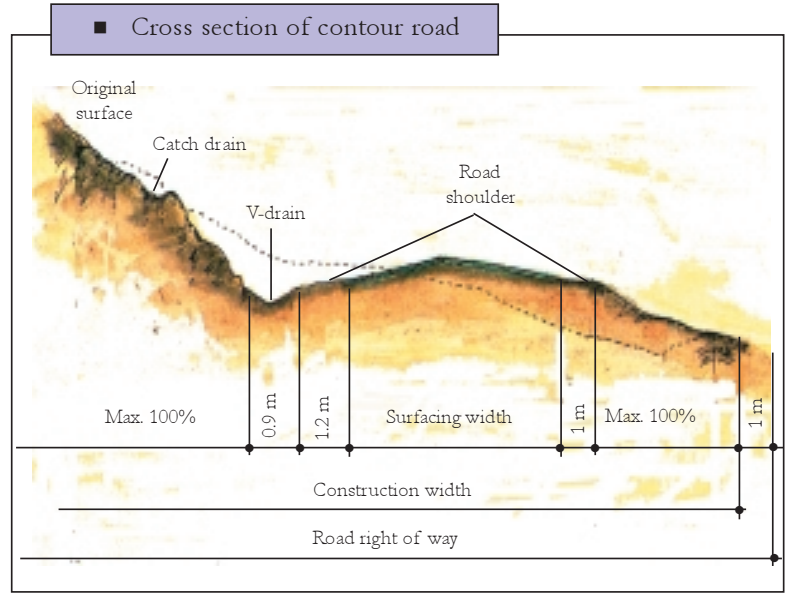

1. Steeper grades (up to $20 \%$ ) for short sections (maximum $500 \mathrm{~m}$ ) would be acceptable if it reduces soil disturbance from road construction

2. Any two sections of road at absolute maximum gradient should be separated by $100 \mathrm{~m}$ of level, or lower-gradient road 
- Road grade specification

\begin{tabular}{|l|c|c|c|}
\hline Road Class & $\begin{array}{c}\text { Maximum } \\
\text { Allowable } \\
\text { Grade }(\%)\end{array}$ & $\begin{array}{c}\text { Maximum } \\
\text { Adverse } \\
\text { Grade }(\%)\end{array}$ & $\begin{array}{c}\text { Length at } \\
\text { Maximum } \\
\text { Grade }(\mathrm{m})\end{array}$ \\
\hline Main road & 10 & 8 & 1000 \\
\hline Branch & 15 & 10 & 750 \\
\hline Feeder road & 18 & 12 & 600 \\
\hline
\end{tabular}

- Road width specification

Maximum Road Width

\begin{tabular}{|l|c|c|}
\cline { 2 - 3 } \multicolumn{1}{c|}{} & \multicolumn{2}{c|}{ Maximum Road Width } \\
\cline { 2 - 3 } \multicolumn{1}{c|}{} & Gravel Surface $(\mathrm{m})$ & Compacted Clay Surface $(\mathrm{m})$ \\
\hline Main road (2-way) & 10.0 & 15.0 \\
\hline Main road (1-way) & 6.3 & 11.0 \\
\hline Branch road & 6.0 & 7.3 \\
\hline Feeder road & 5.0 & 6.0 \\
\hline
\end{tabular}


- Road off-trailing specification

\begin{tabular}{|c|c|}
\hline $\begin{array}{c}\text { Radius } \\
(\mathrm{m})\end{array}$ & $\begin{array}{c}\text { Off-Trailing } \\
(\mathrm{m})\end{array}$ \\
\hline 25 & 1.65 \\
\hline 30 & 1.20 \\
\hline 40 & 0.95 \\
\hline 50 & 0.80 \\
\hline 60 & 0.70 \\
\hline
\end{tabular}

\begin{tabular}{|c|c|}
\hline $\begin{array}{c}\text { Radius } \\
(\mathrm{m})\end{array}$ & $\begin{array}{c}\text { Off-Trailing } \\
(\mathrm{m})\end{array}$ \\
\hline 75 & 0.55 \\
\hline 100 & 0.40 \\
\hline 150 & 0.35 \\
\hline 200 & 0.20 \\
\hline
\end{tabular}

- Curve widening will be required on corners to allow for off-trailing of trailers

- Off-trailing on curves based on a vehicle with overall length of $14 \mathrm{~m}$

- Additional width is required in each lane, i.e. a twolane road requires a total widening of the roadway width to twice the tabulated value 
- Road curves

\begin{tabular}{|l|c|c|c|}
\cline { 2 - 4 } \multicolumn{1}{c|}{} & \multicolumn{3}{c|}{ Design Speed } \\
\cline { 2 - 4 } \multicolumn{1}{c|}{} & $30 \mathrm{kph}$ & $50 \mathrm{kph}$ & $80 \mathrm{kph}$ \\
\hline Minimum radius (use may require signs) & $25 \mathrm{~m}$ & $30 \mathrm{~m}$ & $55 \mathrm{~m}$ \\
\hline Desirable minimum radius & $35 \mathrm{~m}$ & $75 \mathrm{~m}$ & $140 \mathrm{~m}$ \\
\hline Minimum sight distance requaired & $30 \mathrm{~m}$ & $64 \mathrm{~m}$ & $120 \mathrm{~m}$ \\
\hline Meeting sight distance & $50 \mathrm{~m}$ & $100 \mathrm{~m}$ & $220 \mathrm{~m}$ \\
\hline
\end{tabular}

- The minimum radius of the curve is related to visibility and speed of vehicles travelling on the road

- Road drain spacing

\begin{tabular}{|c|c|c|}
\cline { 2 - 3 } \multicolumn{1}{c|}{} & \multicolumn{2}{c|}{ Maximum Drain Spacing $(\mathrm{m})$} \\
\hline Centerline Gradient (\%) & Unstable Soil & Stable Soil \\
\hline $0-15$ & 40 & 60 \\
\hline $16-20$ & 20 & 40 \\
\hline $21-25$ & 10 & 20 \\
\hline
\end{tabular}

- Drain all roads to minimize sediment production

- Road drains should be constructed as follows:

* At changes of slope

* Within 50 metres of watercourse crossings

* Additional drains to meet the maximum spacing requirement 


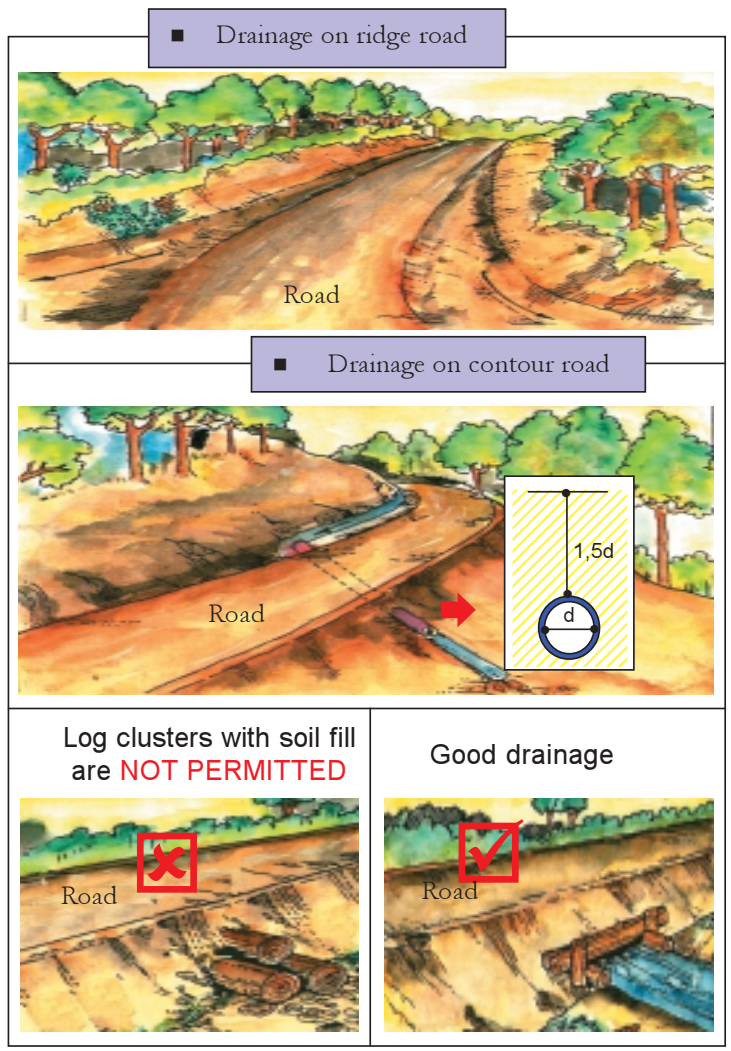




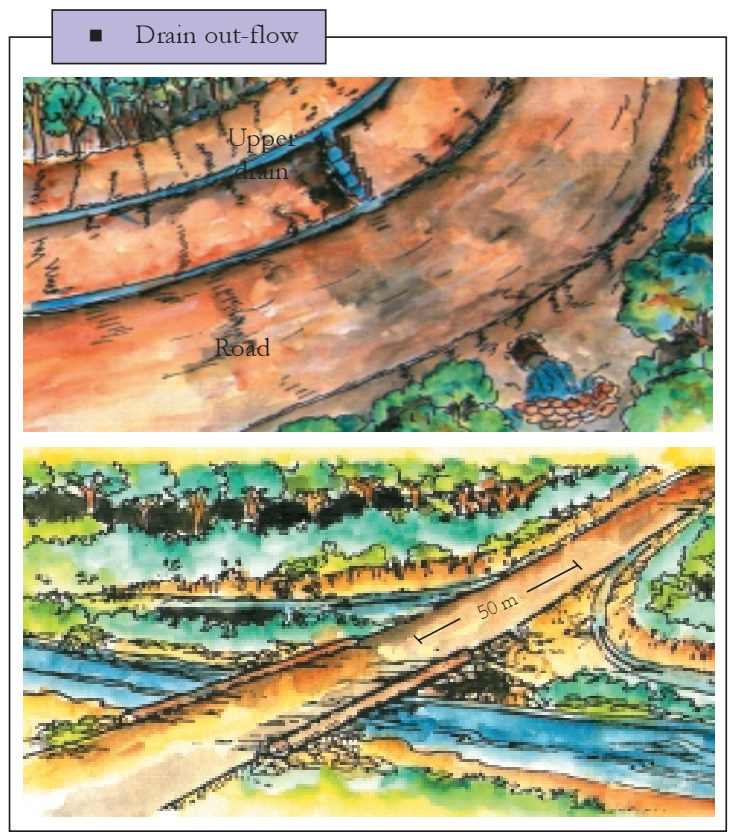

- Drain out-flow should be diverted into surrounding vegetation at least $50 \mathrm{~m}$ before a watercourse 

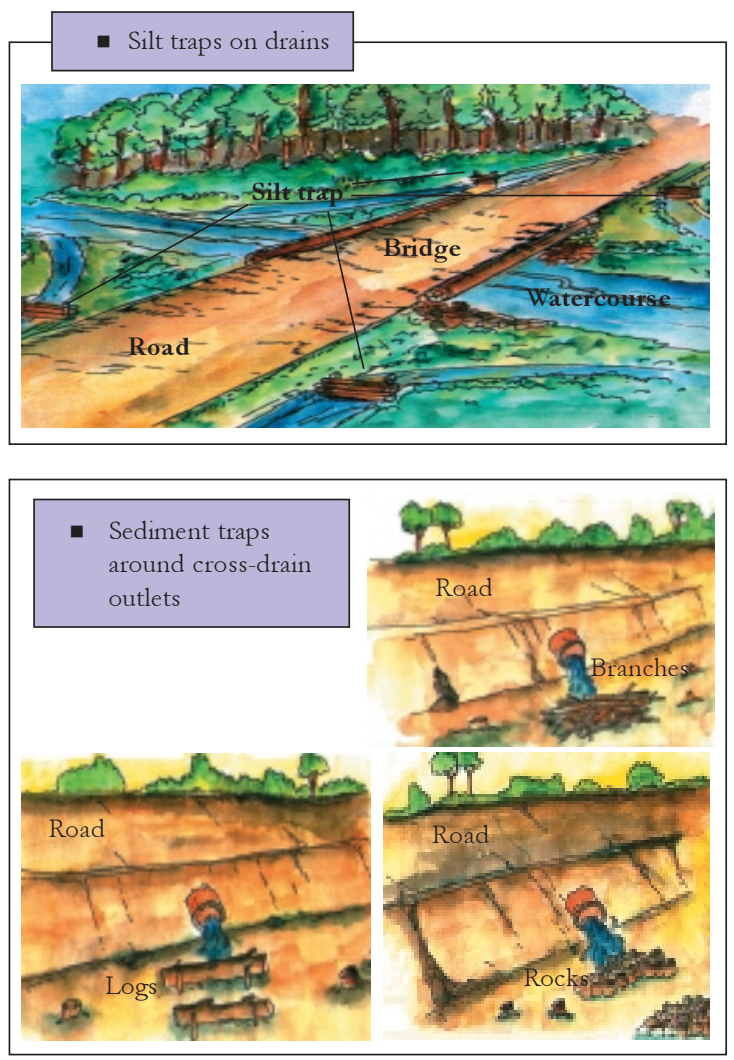


\section{Measures to minimize erosion on batter slope

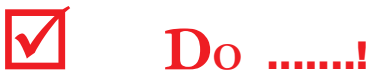

- Planted vegetation on batter slope
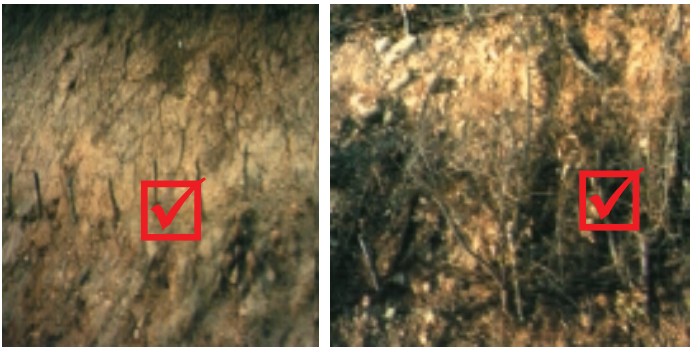

- Sediment trap at roadside drain

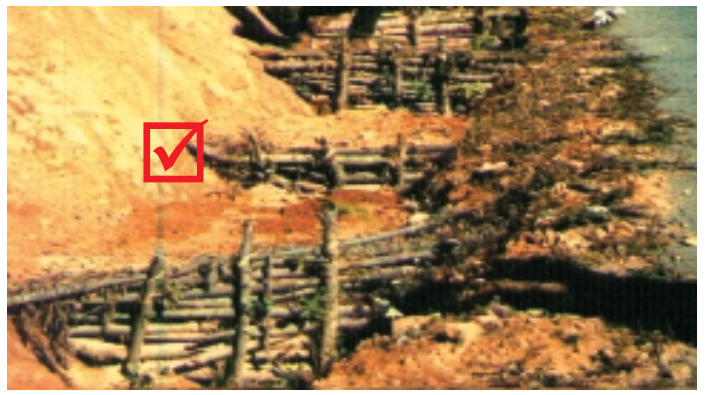


- Road construction

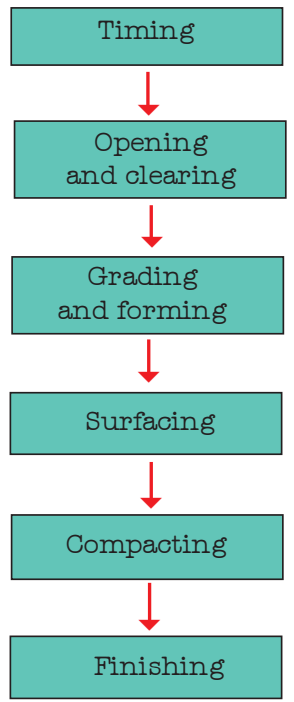




\section{Timing}

Road construction should be conducted one year before logging and should not take place during the wet season.

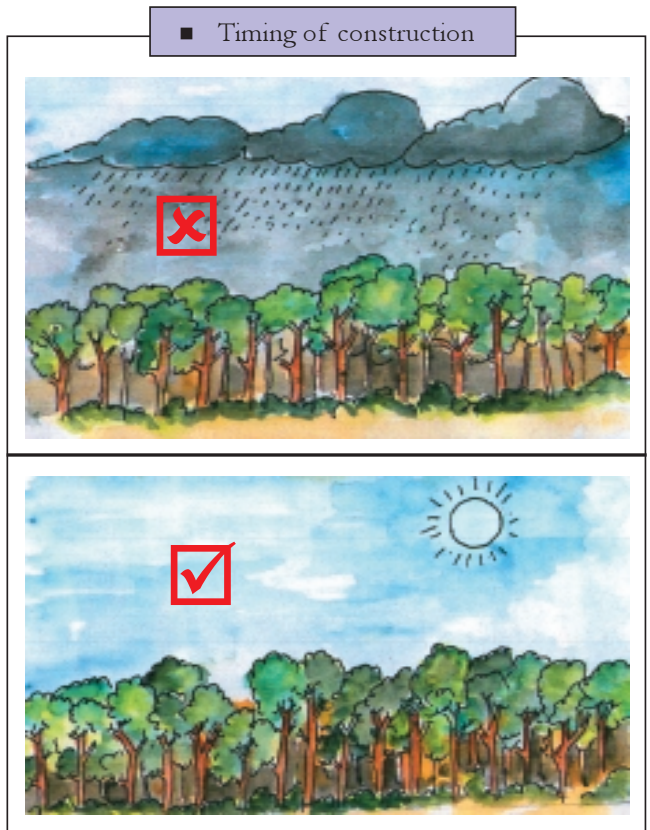




\section{Opening and clearing}

Removal of trees, stumps, roots and organic matter from the road construction area.
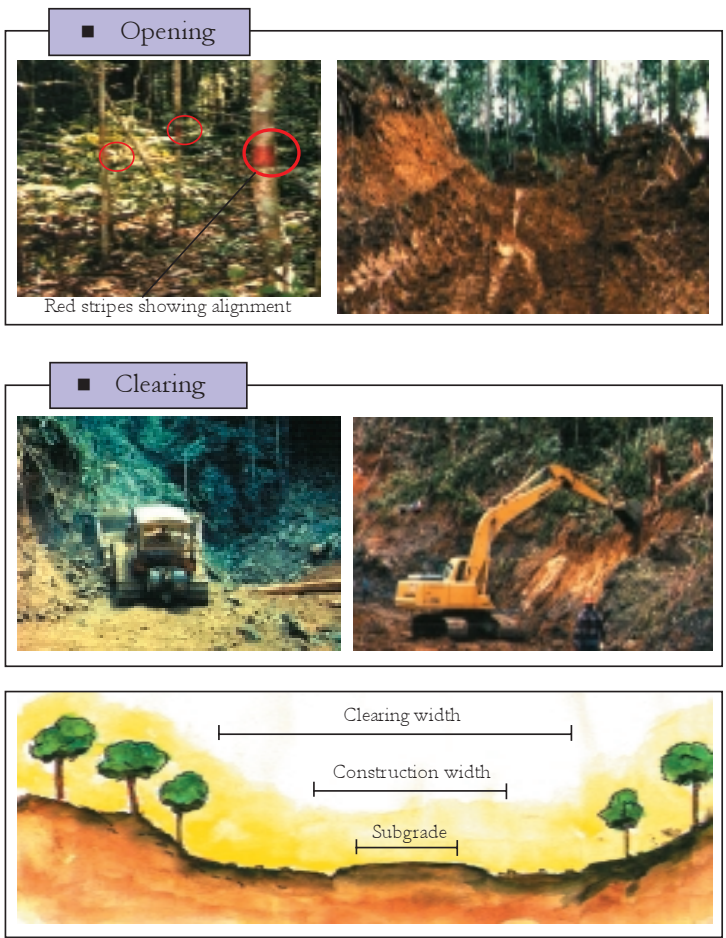


\section{Grading and forming}

Grading and forming of the road is undertaken after area has been opened and cleared. Earthmoving is done by bulldozers over short distances. Dump trucks and loaders are used for longer distances. Bulldozers and motor graders are the basic equipment used for grading and forming.

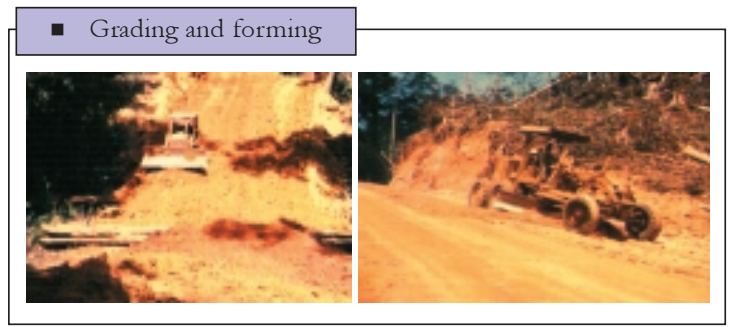

\section{Surfacing}

All-weather forest roads require a surface application of rock, gravel or acceptable surface material. This material is usually brought from local quarries. The material is hauled by dump trucks and spread with motor graders.

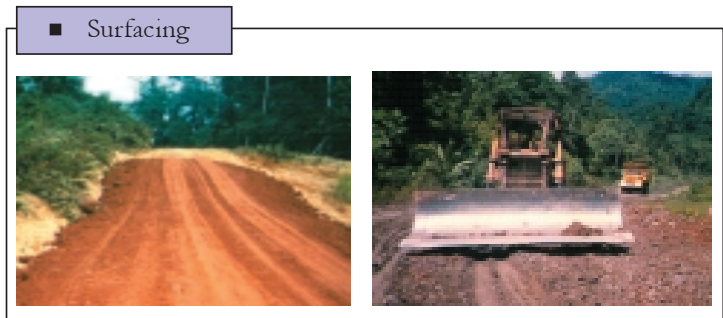




\section{Compacting}

Compacting the road is important. The vibration roller is the basic equipment needed to compact the soil and roading material.

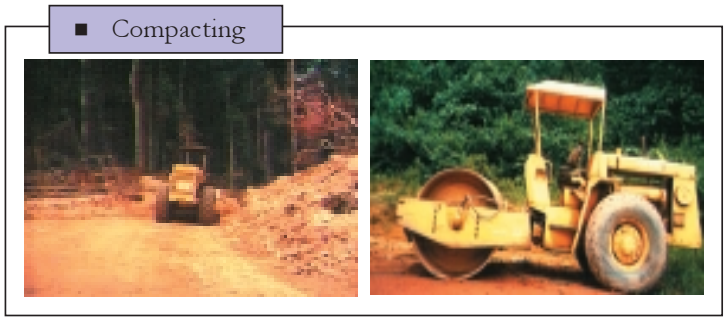

\section{Finishing}

The final step in road construction is the crowning, sloping of shoulders and slopes and ditching. This is usually accomplished with a motor grader.

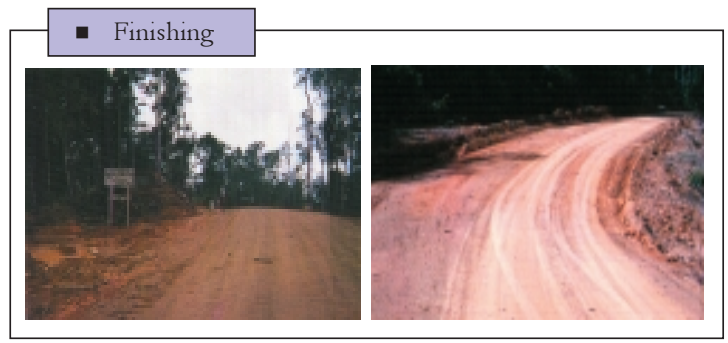




\section{- Watercourse crossings}
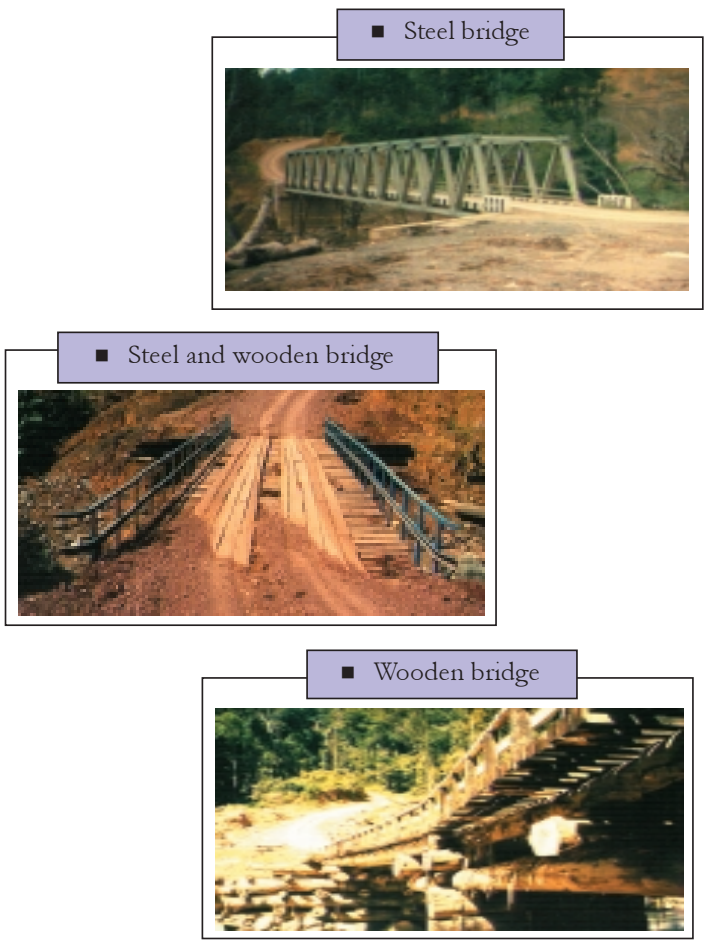


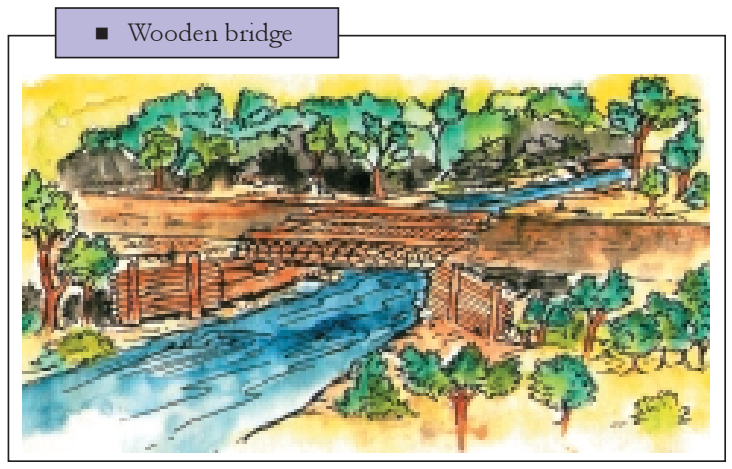

\begin{tabular}{|c|c|c|}
\hline In fresh water & In salt or brackish water & Above water level \\
\hline $\begin{array}{l}\text { Balau (Dipterocarpus } \\
\text { mundus V.SI.) }\end{array}$ & $\begin{array}{l}\text { Belian (Eusideroxylon } \\
\text { zwageri T.et.B) }\end{array}$ & $\begin{array}{l}\text { Balau (Diptercocarpus } \\
\text { mundus V.SI.) }\end{array}$ \\
\hline $\begin{array}{l}\text { Bangkirai (Shorea } \\
\text { laevis Ridl.) }\end{array}$ & Resak (Vatica spp.) & $\begin{array}{l}\text { Bangkirai (Shorea } \\
\text { laevis Ridl.) }\end{array}$ \\
\hline $\begin{array}{l}\text { Belian (Eusideroxylon } \\
\text { zwageri T.et.B) }\end{array}$ & Keranji (Dalium spp.) & $\begin{array}{l}\text { Belian (Eusideroxylon } \\
\text { zwageri T.et.B) }\end{array}$ \\
\hline Chengal (Hopea spp.) & & Chengal (Hopea spp.) \\
\hline $\begin{array}{l}\text { Giam (Vatica } \\
\text { flavovirens V.SI) }\end{array}$ & & $\begin{array}{l}\text { Giam (Vatica } \\
\text { flavovirens V.SI) }\end{array}$ \\
\hline \multirow[t]{3}{*}{ Keranji (Dialium spp.) } & & Keranji (Dialium spp.) \\
\hline & & $\begin{array}{l}\text { Kempas (Koompassia } \\
\text { malaccensis Maing) }\end{array}$ \\
\hline & & $\begin{array}{l}\text { Merbau (Intsia bijuga } \\
(\text { Colebr. }))\end{array}$ \\
\hline
\end{tabular}



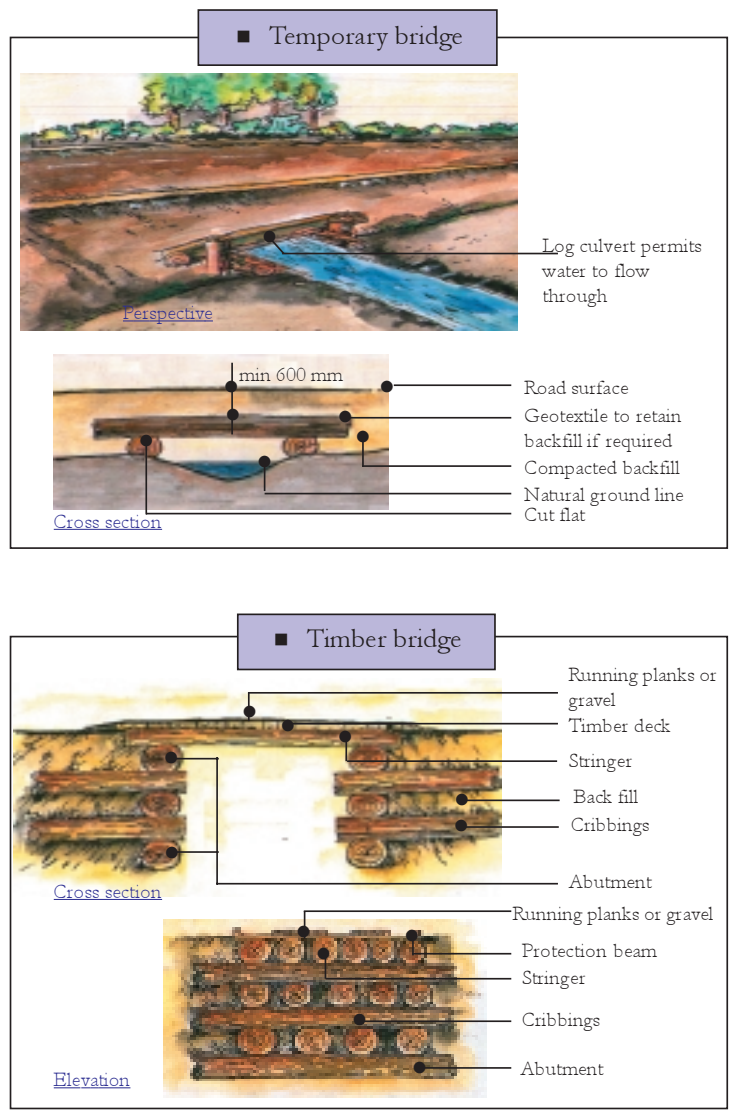


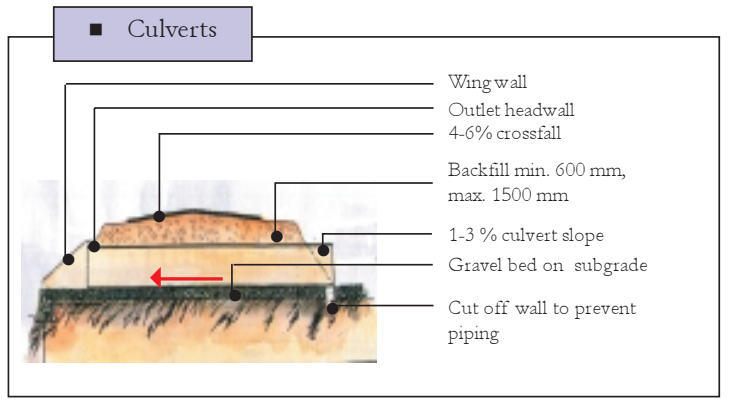




\section{Pre-logging Field Preparation}

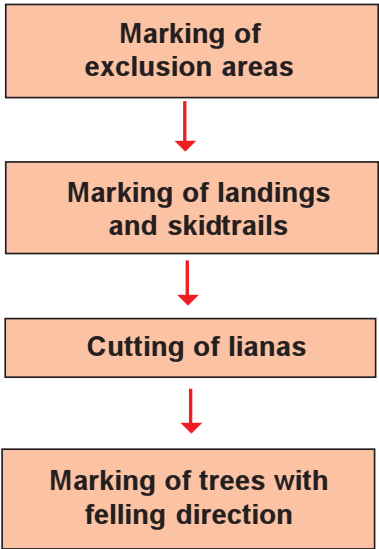




\section{- Marking of exclusion areas}

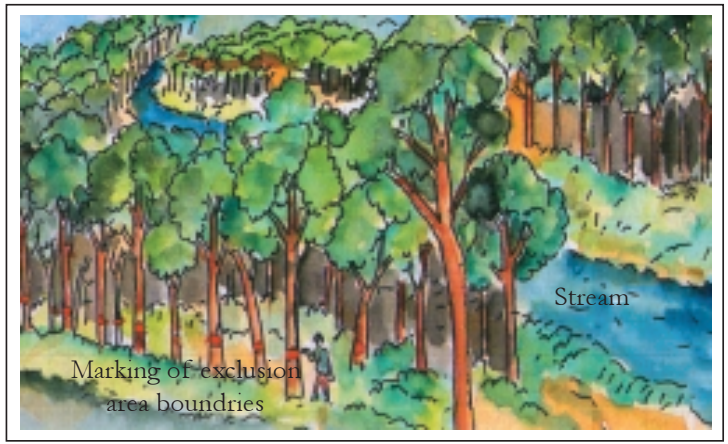

\begin{tabular}{|l|l|}
\hline \multicolumn{2}{|c|}{ " Buffer zone marking } \\
\hline Category & Field Marking \\
\hline Buffer zones & $\begin{array}{l}\text { Perimeter with an "inverted T" as high as } \\
\text { possible on one side facing the logging area }\end{array}$ \\
\hline
\end{tabular}




\section{- Marking of landings and skidtrails}

\begin{tabular}{|l|l|}
\hline \multicolumn{1}{|c|}{ Skidtrail marking } \\
\cline { 2 - 2 } \multicolumn{1}{|c|}{ Category } & \multicolumn{1}{|c|}{ Feild Marking } \\
\hline Major skidtrail & $\begin{array}{l}\text { Central line with 1 vertical stripe as high as possible, } \\
\text { front and back of trees, spaced at approx. } 10 \mathrm{~m} \text { intervals }\end{array}$ \\
\hline Major skidtrail ends & $\begin{array}{l}\text { Marked with 2 parallel stripes as high as possible facing } \\
\text { the track }\end{array}$ \\
\hline Minor skidtrail & $\begin{array}{l}\text { Central line with 1 vertical stripe as high as possible, } \\
\text { front and back of trees, spaced at approx. 10 } \mathrm{m} \text { intervals }\end{array}$ \\
\hline Temporary stream crossings & Marked with $\mathrm{S}$ on the trees on either side of stream \\
\hline
\end{tabular}

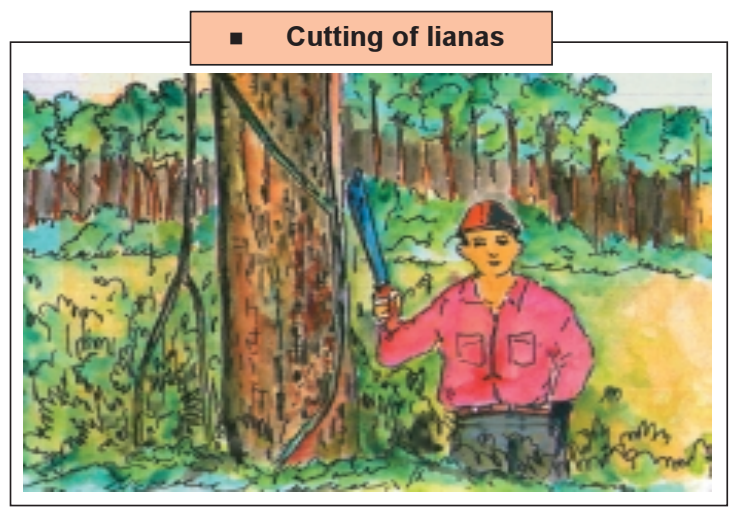




\section{Marking of trees with felling direction}

\section{Tree marking decision chart}

Diameter greater than minimum cutting limit or tree is dead, dying, defective or in area to be cleared for road/landing according to

Silvicultural Prescriptions
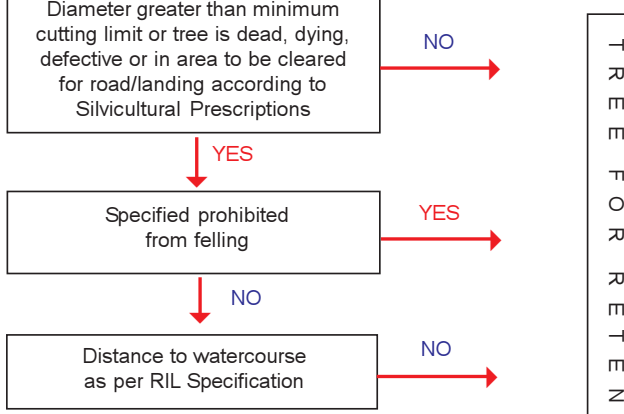

$\downarrow$ YES
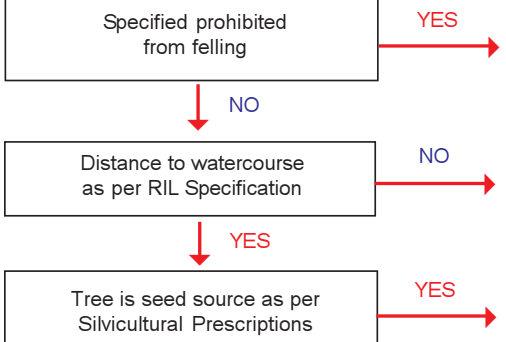

$\downarrow \mathrm{NO}$

\section{MARK TREE FOR FELLING}


- Marking of felling direction

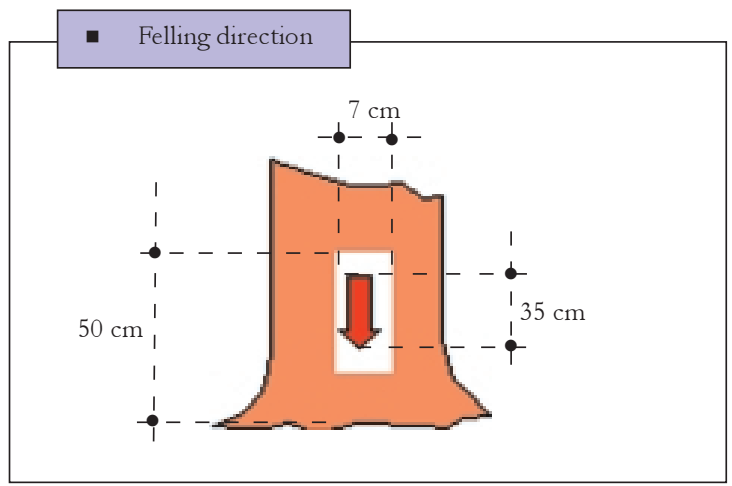




\section{LOGGING OPERATIONS}

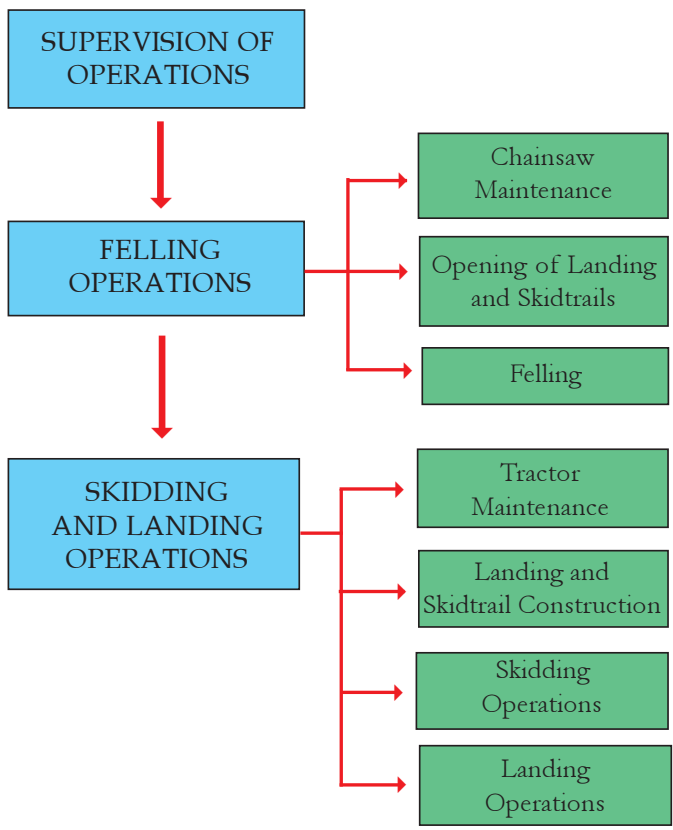




\section{SUPERVISION OF OPERATIONS}

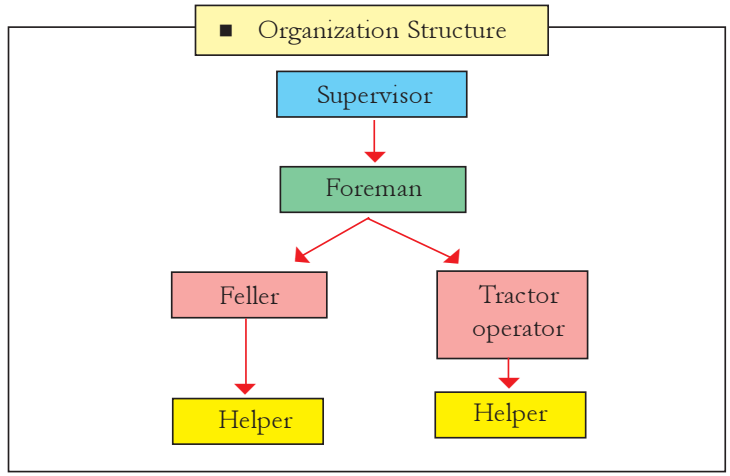

- Job Descriptions

Production Supervisor

- Direct involvement in pre-logging planning

- Train and familiarise staff with the operational and safety requirements

- Coordinate logging operations

- Direct supervision of logging operations

- Maintain and submit accurate records as required by the company

- Render first aid as required

- Arrange rapid evacuation to hospital of any injured worker as required 
- Direct involvement in pre-logging planning

- Monitor progress of felling and skidding activities (incl. providing guidance)

- Check and evaluate results of felling, skidding and post-logging activities

- Report result of the evaluation and appraisal by felling compartment to the Base Camp Manager, Timber Production Division and Forest Planning Division

- Calculate and prepare payment/salary proposal based on tariffs for felling and skidding, to be submitted to the Base Camp Manager

\section{Felling and skidding foremen}

- Ensure that only authorized and adequately trained workers operate machines and equipment

- Coordinate, improve and provide guidance in skidtrail construction, felling and skidding operations

- Control and monitor production process in order to achieve the targeted quality and quantity

- Ensure that all equipment and facilities are properly inspected and maintained 


\section{Feller}

- Maintain chainsaws in a safe operational condition

- Be responsible for felling activities

- Fell each harvestable tree according to the planned or proper felling direction

- Buck the felled trees according to cross-cutting rules and procedures

- Open skidtrail and winching path

- Helper for felling

- Assist feller, especially in informing him about the location of harvestable trees

- Assist in checking whether all trees to be cut have been felled

- Construct safety or escape lines for the feller

- Assist in removing branches from the felled trees and in measuring log lengths for bucking at the felling site

- Mark stumps left by the felled trees with tree number as well as logs produced with log numbers

- Carry chainsaw bars and chains, maintenance tools and lubricating oil

- Carry food and drinking water

- Clean chainsaw and all other tools 
- Be responsible for the tractor and its accessories

- Construct landings and skidrails

- Adhere to technical standards of skidding as planned

- $\quad$ Conduct preventive measures against postharvest damage to residual stands and the environment

- $\quad$ Be responsible for skidding activities

- Helper for Skidding

- Assist the tractor operator in the maintenance of tractor

- Be responsible for choker/hooks and cables

- Locate logs to be skidded

- Install chokers/hooks for winching

- Direct the tractor operator to the location of logs to be skidded

- $\quad$ Pull winch cable towards logs to be winched and attach it to the chokers/hooks

- $\quad$ Signal the tractor operator to start winching

- Assist the tractor operator in taking measures to prevent further post-harvest damage 
- Information and discussion on logging plan and operations

All crew members involved should know their duties and responsibilities, expected work procedure and standards

\section{'Morning Talk' :}

Meeting of skidding/felling foremen, chainsaw operators and tractor operators

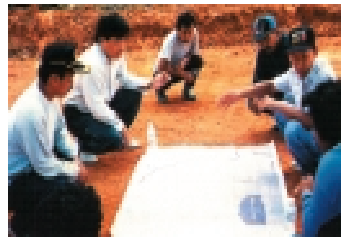

'Weekly Meeting' :

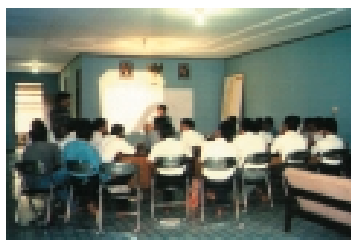

Meeting of production supervisors, block inspectors, skidding/felling foremen, chainsaw operators and tractor operators 


\section{FELLING OPERATIONS}

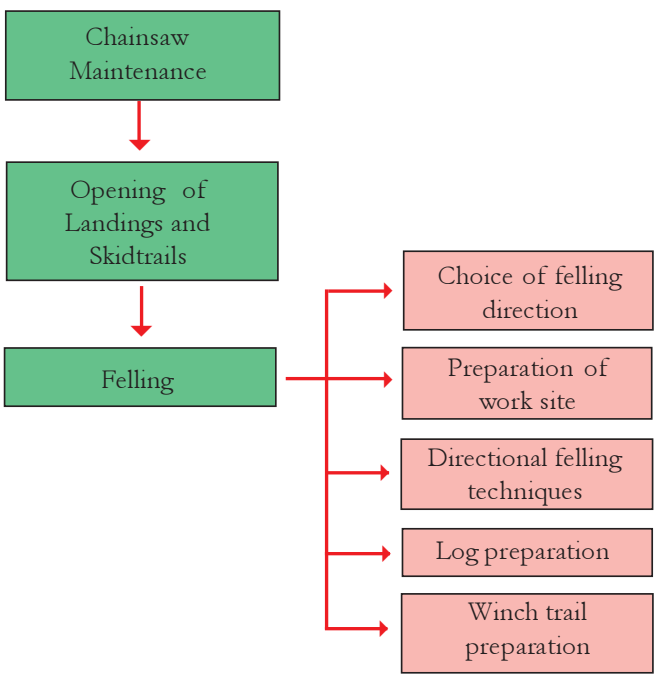




\section{Chainsaw Maintenance}

The following parts of chainsaw are to be checked daily:

* Saw chain

* Guide bar

* Gears

* Oil filter and fuel filter

* Spark plugs

* Exhaust system

* Chain brake and front handle guard
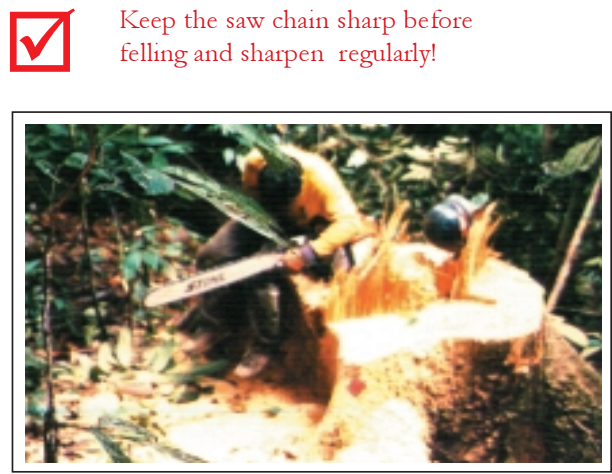


\section{Opening of Landing and Skidtrails}

* Landing and skidtrail openings should be set up prior to felling

* Tools to be used: logging plan and chainsaw

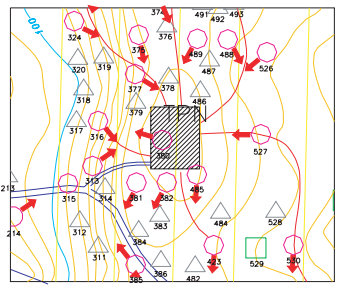

1. Landing and skidtrail network map

2. Felling to open the landing and skidtrails (Et-0)

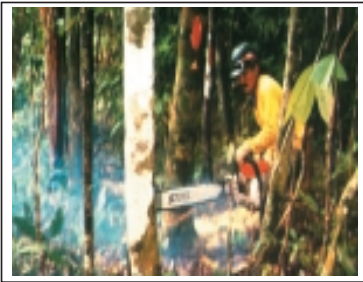

$* \mathrm{Et}=$ Time of logging (year); $(-)=$ Before logging 
1. The team required for opening landings and skidtrails consists of a chainsaw operator and a helper

2. Chainsaw operators open landing areas and skidtrails by cutting all trees with $\varnothing \geq 15 \mathrm{~cm}$ within these areas

3. The felling begins from the end of the prospective skidtrail to the landing with felling direction away from the landing

4. Undercuts and backcuts are made as low as possible, with felling directions coinciding with the skidtrail direction or right on the skidtrail 


\section{F elling}

- Felling procedure

1. The Felling commences according to the felling pattern on the map

2. Check felling condition, determine felling direction, prepare work site, open escape routes and caution all persons in vicinity of felling area (danger zone)

3. Make the notch and the backcut on the stump as low as possible

4. Top and limb the fallen tree

5. Clear buttresses from the stem

6. Measure and buck stem according to specifications

7. Place tree number on stump and on the bottom of each log section

8. Open winch trail

9. Proceed to other harvestable trees

\section{AVOID .....!}

- Trees falling across rivers or in Protected Areas

- Damage to potential crop trees and protected trees 


\section{Choice of felling direction}

- Felling direction

Procedures:

1. The best felling direction is towards or away from skidtrails at an angle of $30^{\circ}-45^{\circ}$ (herringbone pattern), or the tree should fall in a parallel position on the skidtrail away from the skidding direction

2. If possible, the felled tree should be directed to an open site. Preferably other trees should be felled into the same gap (maximum 3)

3. On steep areas, the felling direction should be towards the side of the slope (along the contour)

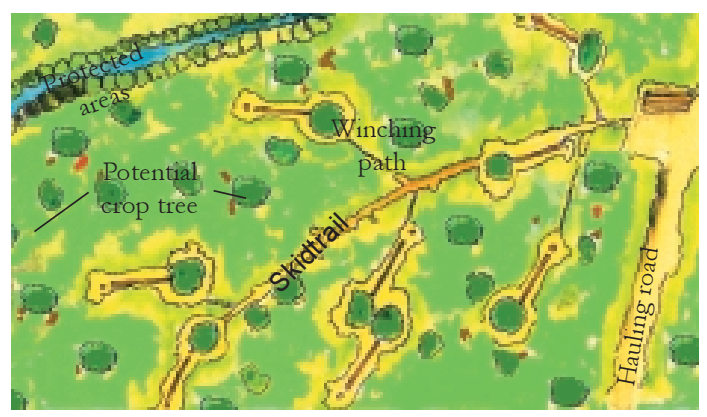


Preparation of work site
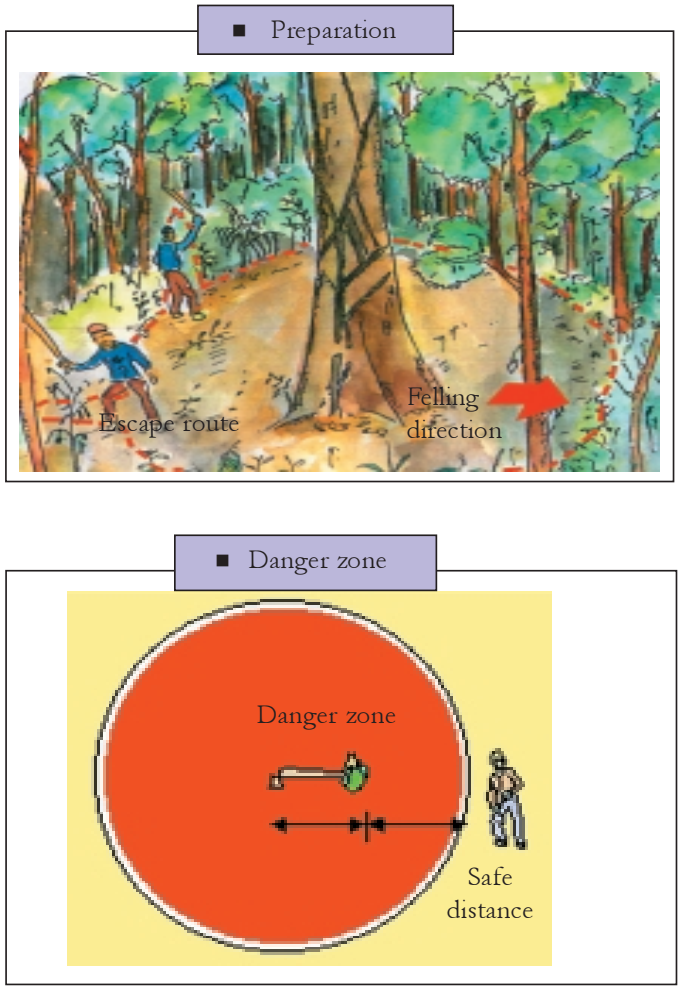
Directional felling techniques

The use of felling wedges is recommended to give additional control over felling direction

- Felling technique for leaning trees
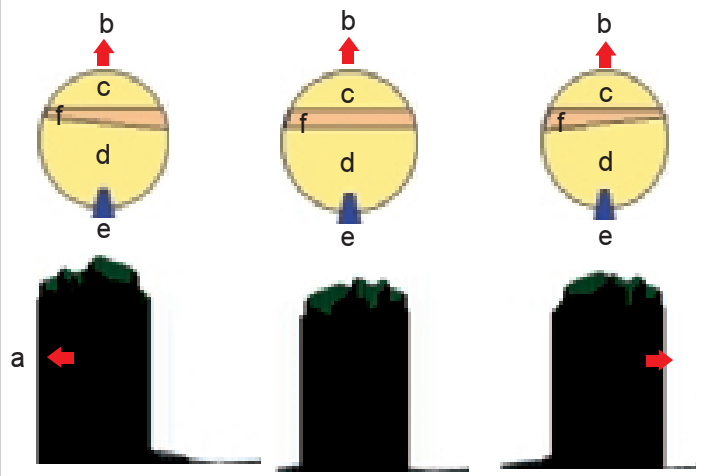
$\mathrm{a}=$ Tree leaning direction $\mathrm{d}=$ Backcut
$\mathrm{b}=$ Felling direction
$\mathrm{e}=$ Wedge
$\mathrm{c}=$ Undercut
$\mathrm{f}=$ Hinge

Directional felling for leaning tree:

- Notch is cut according to expected felling direction

- Create asymmetric hinge, stronger end opposite the leaning direction

- Use of felling wedges to give additional control over felling direction 


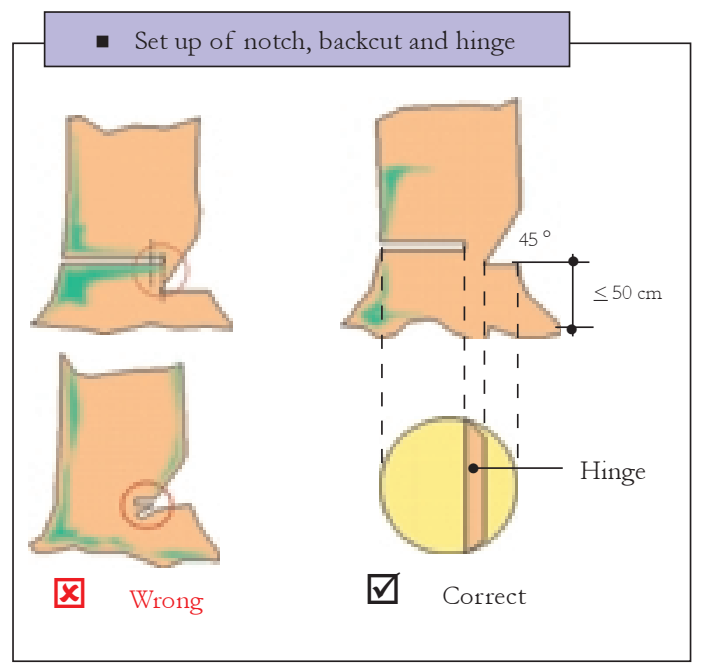

\section{- Cutting technique of backcut}

- Small tree

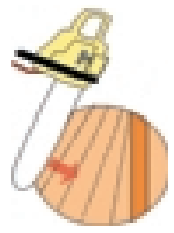

- Big tree

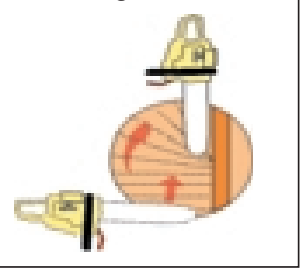




\section{- Felling technique for normal tree}

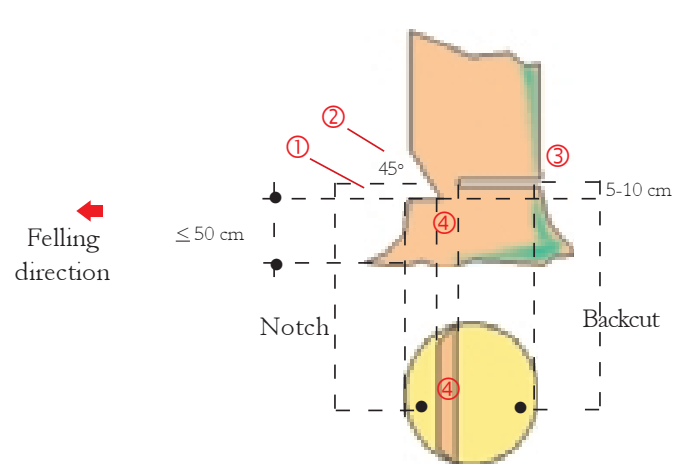

Work steps:

(1) The first cut is a horizontal cut as deep as 1/4$1 / 3$ of tree $\phi$ at a maximum height of $50 \mathrm{~cm}$

(2) The second cut is a slanting cut, making an angle of $45^{\circ}$ towards the end of the horizontal cut

(3) The third cut is a backcut. It is made about $5-10 \mathrm{~cm}$ above and on the opposite side of the horizontal cut

(4) Leave a hinge of $1 / 10$ to $1 / 6$ of the tree $\phi$ 
- Felling technique for large tree

\section{Create notch}

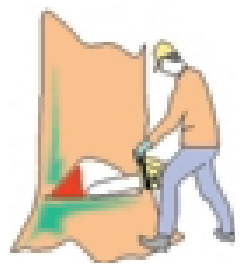

2. Make hole at the center

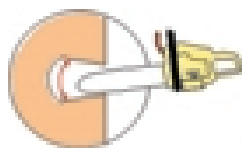

3. Backcut $10-20 \mathrm{~cm}$ above the undercut of the notch

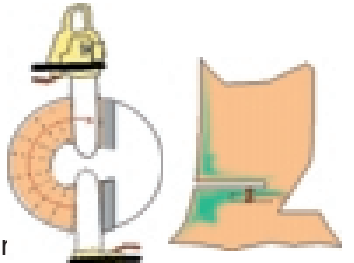

- Utilization technique of stem with buttresses

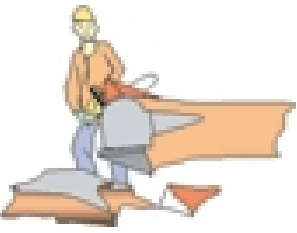

Clean the buttresses after tree is felled

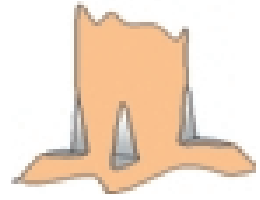

Large buttresses are cut before felling the tree 


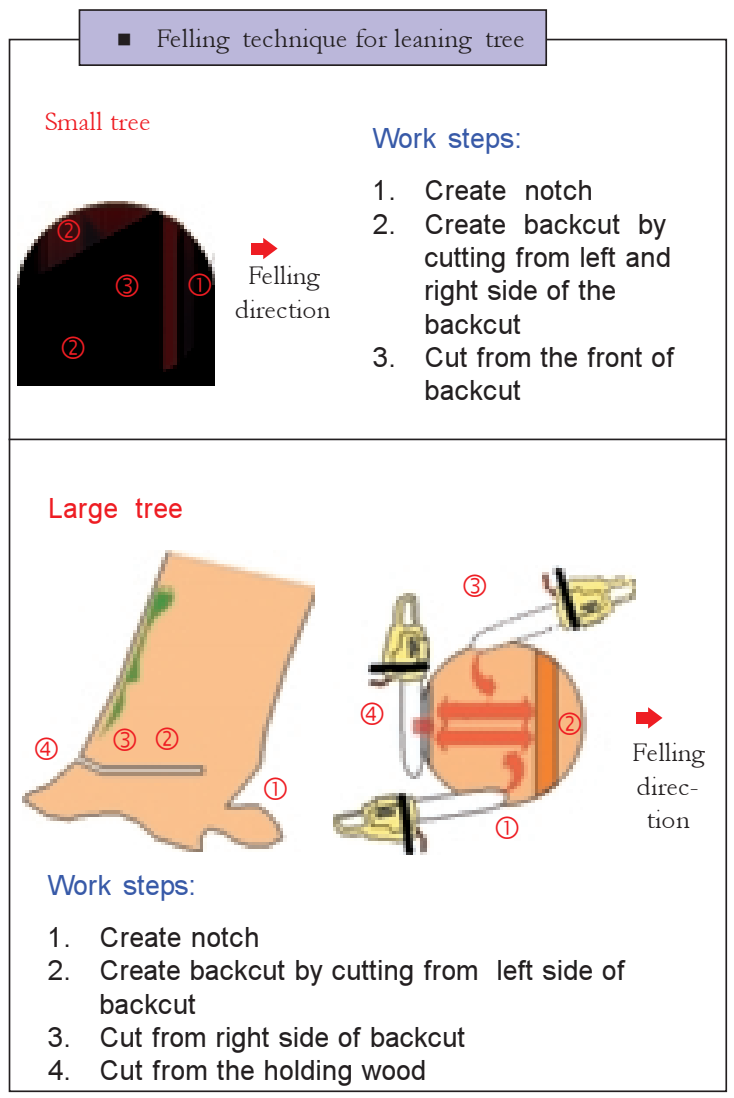


- Felling technique for trees with buttresses
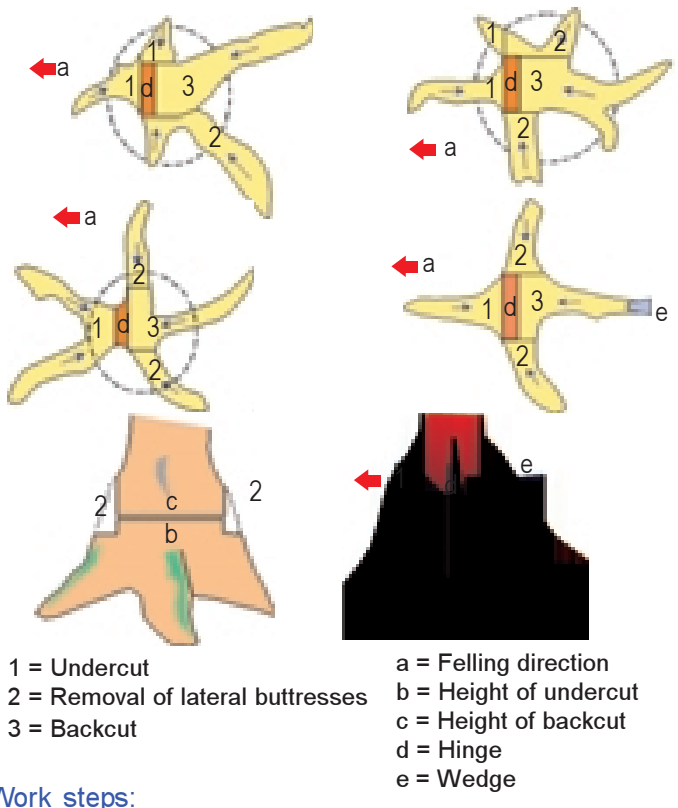

Work steps:

$1=$ Undercut

$2=$ Removal of lateral buttresses

$3=$ Backcut

1. Create notch

2. Remove buttresses on the right and left side of backcut

3. Create backcut 


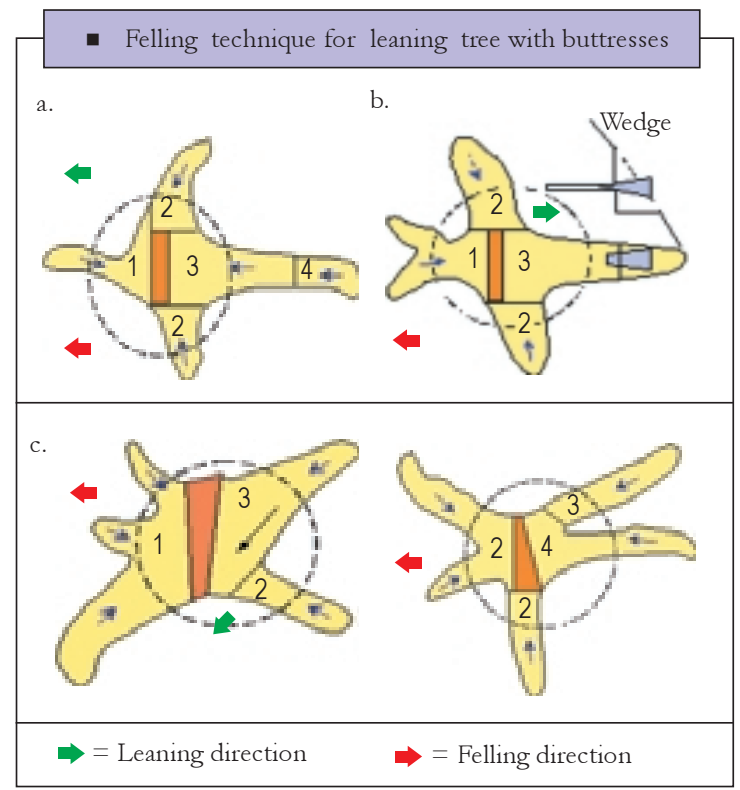

a. The tree is leaning in the felling direction

b. The tree has to be felled against the lean

c. The tree has to be felled slanting to the direction of lean

Numbers 1, 2, 3, 4 indicate sequence of work (prepare notch, remove the lateral buttresses, backcut and cut the supporting buttress) 
- Log preparation

1. Stem should be measured and its log sections should be determined before cutting the stem. Cross-cuts are no more than $10^{\circ}$ from vertical

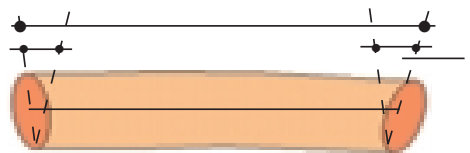
must be less than $10^{\circ}$

2. Cutting of tension stems

a. Cut on the compression side

b. Cut on the tension side

a.

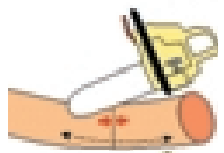

a.

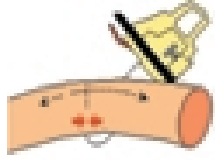

b.

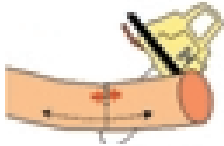

b.

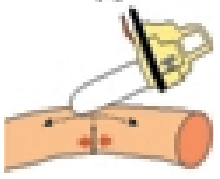

3. Stem cutting technique

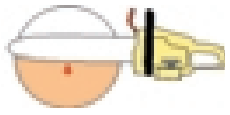

$1+$

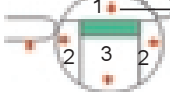

Compression zone

Vertical cut 
Winch trail preparation

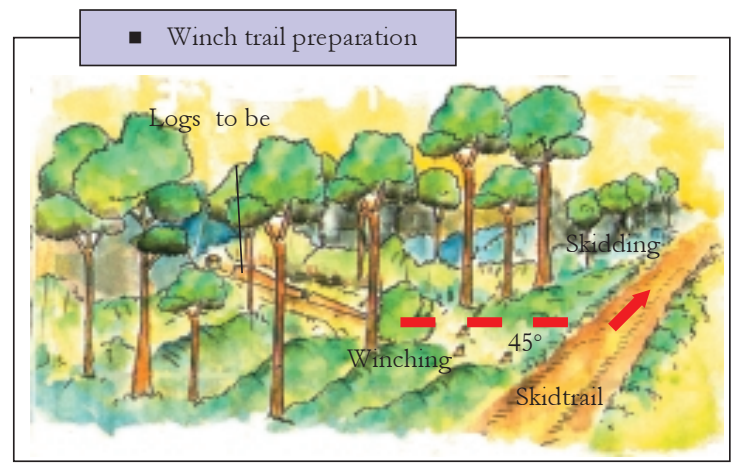




\section{SKIDDING AND LANDING OPERATIONS}

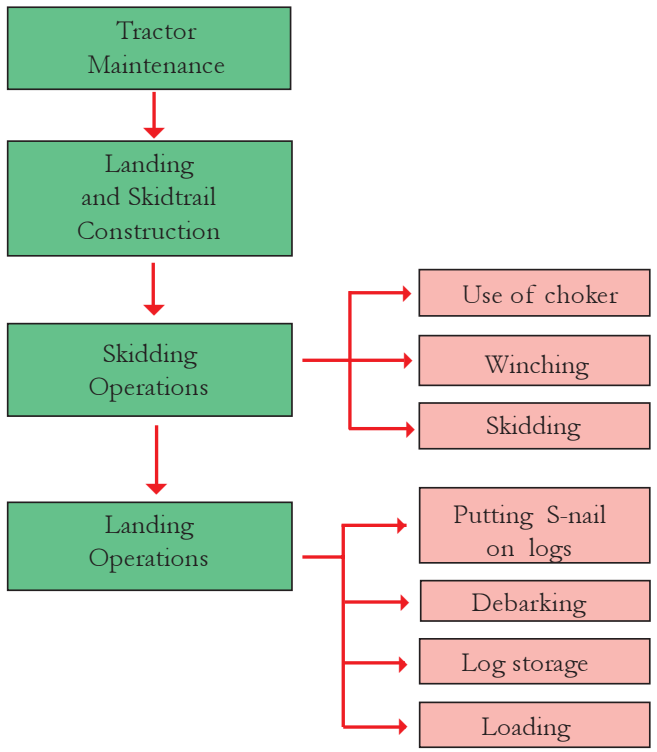




\section{Tractor Maintenance}

Tractor maintenance should be undertaken by the tractor operator and his helper every morning before operating and every afternoon

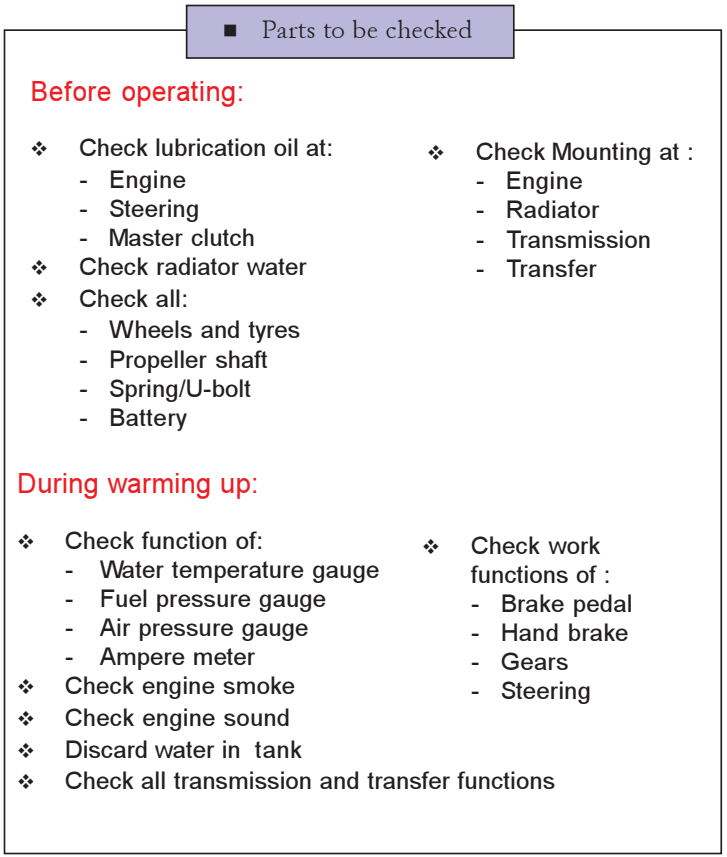




\section{After operating:}

- Fill fuel tank on level ground

- Park the tractor

- Switch off machine after 5 minutes

- Clean up inside cabin

- Clean dirt off wheels and tyres

- Clean dirt off lamps

- Ensure cab doors are securely closed

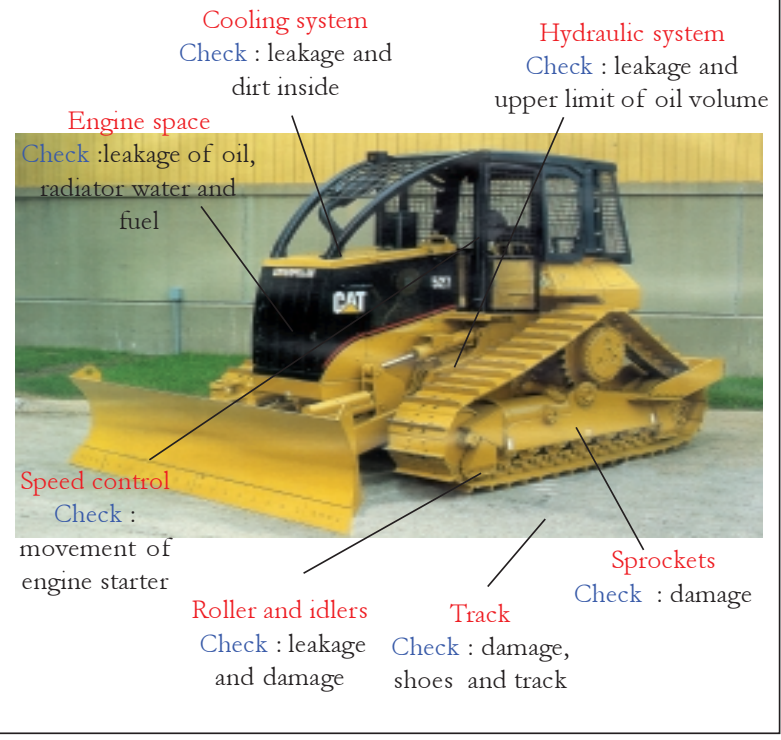




\section{Landing and \\ Skidtrail Construction}

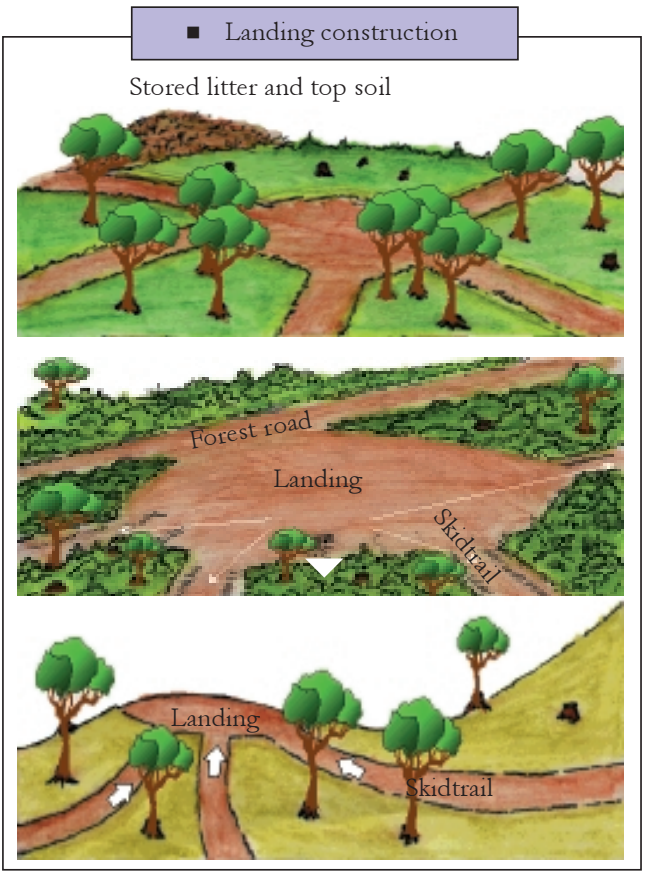

- Size of landing: $900 \mathrm{~m}^{2}$

- Drains should be constructed and must empty on to stable vegetated areas 


\section{Skidtrail construction}

Steps for skidtrail construction:

Et-1*

Et-1

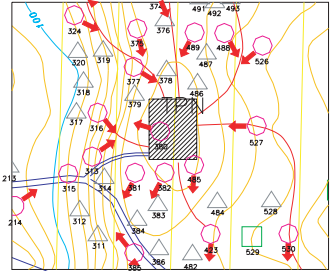

Skidtrail network map

Et-0

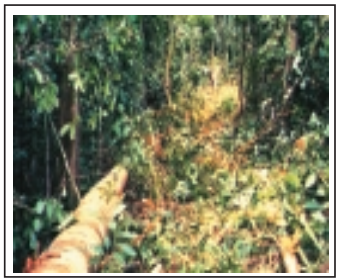

Skidtrail construction

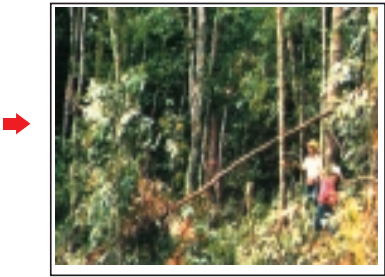

Skidtrail marking

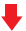

Et-0

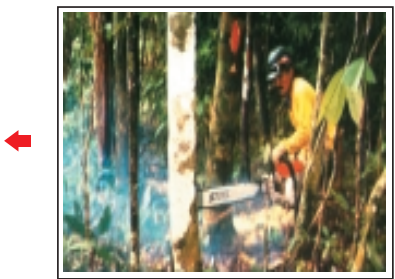

Skidtrail opening

$*$ Et $=$ Time of logging (year); $(-)=$ Before logging 
- Skidtrail construction begins with skidding team and tractor after completion of skidtrail opening and felling activities

- To finish up the skidtrail construction simply remove trees already cut (surface is not to be disturbed)

- Avoid cutting and filling

- Small stems and branches are to remain on skidtrail surface to help soil protection

\section{Skidding Operations}

\section{Skidtrail procedure}

- Skidding operations commence after the construction of the skidtrail

- The helper sets the choker cable/hook on the log

- The helper gives signals to the tractor operator to assist winching operation

- The helper pulls the winch cable out and hooks the choker cable on the log to be winched

- The helper moves to a safe place and gives the signal to start winching

- During winching the tractor should remain on the skidtrail

- After winching, the log is skidded along the skidtrail to the landing

- The helper looks for other log(s) consulting the Logging Map 


\section{AVOID ...!}

凶

1. Skidding during rain and when soil is still wet

2. The tractor moving off the skidtrail looking for logs to be skidded

3. The blade contacting the soil surface and standing trees during skidding activities

4. The tractor moving into protected areas (exclusion zones)

\section{Skidtrail equipment}

- Logging plan map from felling team

- Tractor equipped with winch, blade $<4$ meter (wide)

- 2-4 cable chokers/hooks

- 1 block

- 2 pairs of boots/shoes

- 2 helmets

- 2 pairs of gloves

- 2 sets of work clothing

- 2 food caters and 5 litres of drinking water 
Use of choker
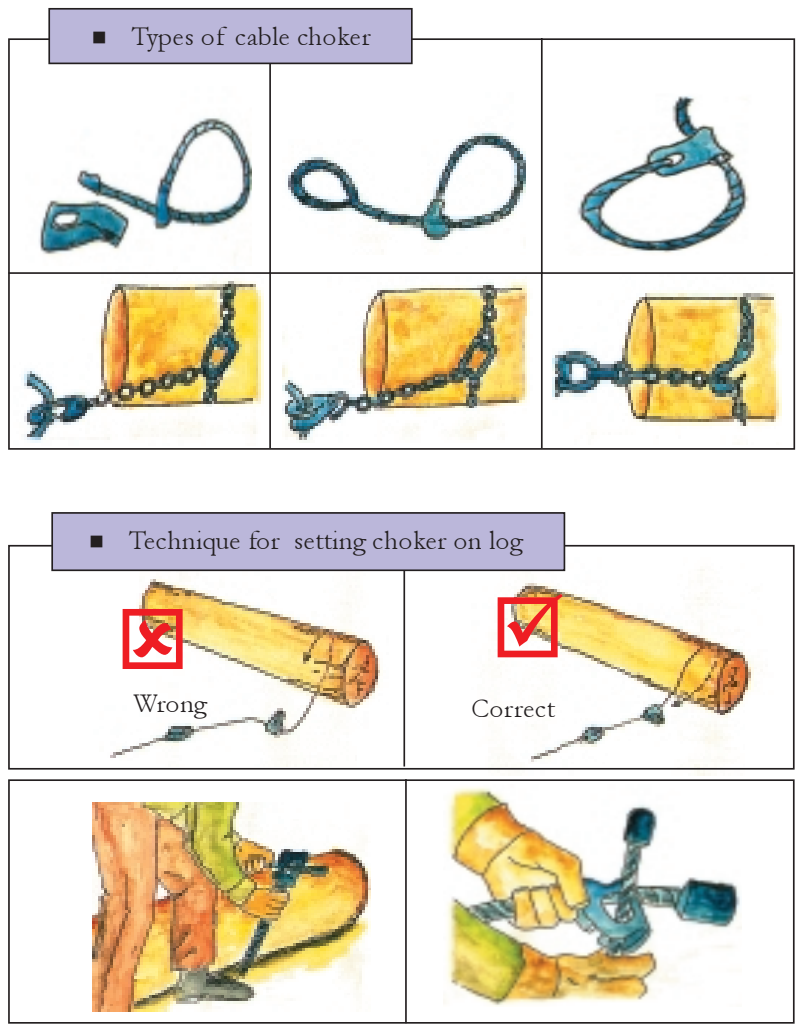


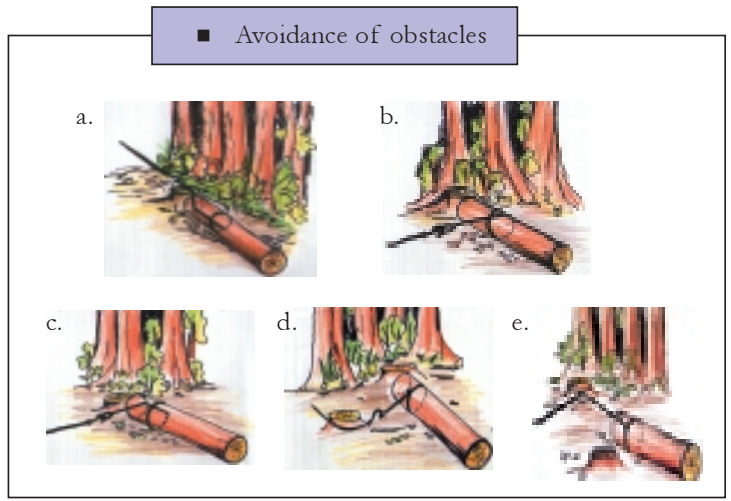

W in ching

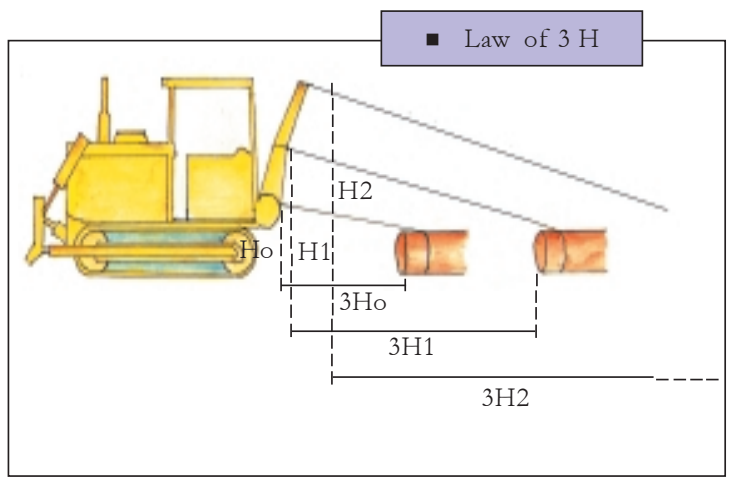


- Chain winching technique

$\begin{aligned} & \text { 1. Tractor reverses } \\ & \text { down hill, then pulls } \\ & \text { the log(s) (tractor } \\ & \text { remains in stationary } \\ & \text { position) }\end{aligned}$
$\begin{aligned} & \text { 2.After the winched } \\ & \text { log(s) come near the } \\ & \text { tractor, the winch } \\ & \text { cable is released }\end{aligned}$
$\begin{aligned} & \text { 4. After the tractor has } \\ & \text { arrived at the landing, } \\ & \text { winching of log(s) } \\ & \text { continues until the } \\ & \text { log(s) reach the } \\ & \text { landing }\end{aligned}$
3. Tractor moves ahead
to the landing (without
pulling the log(s)


Skid ding

- Skidding technique to minimize damage

- Tractor blade should be raised when skidding

- Use arch to lift the end of the log off the ground

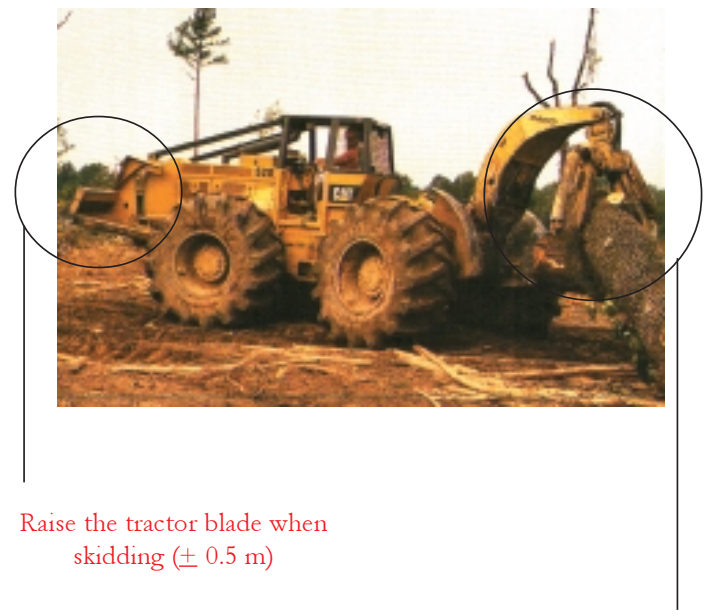

Use arch to lift the end of the $\log$ 
- Hand signals for skidding

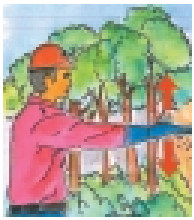

a. Slacken the winch cable!

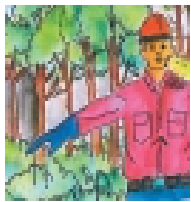

d. Stop tractor!

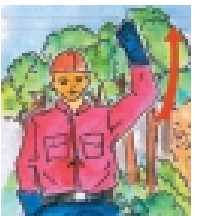

b. Winching

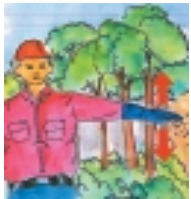

e. Tractor may start winching

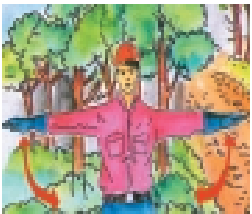

c. Stop winching and hold

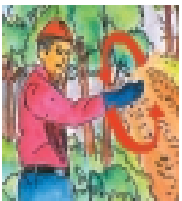

f. Back up tractor!

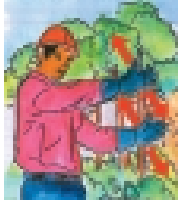

g. Drop chokers here!

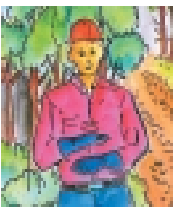

h. Use upper winch!

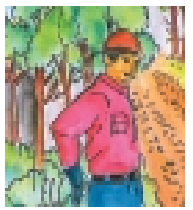

i. Go back inside tractor!

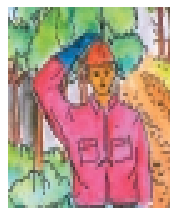

j. Move forward! 
- Use shunt skidding method for turning logs

The shunt skidding method is applied to reduce disturbance of the residual stand and soil
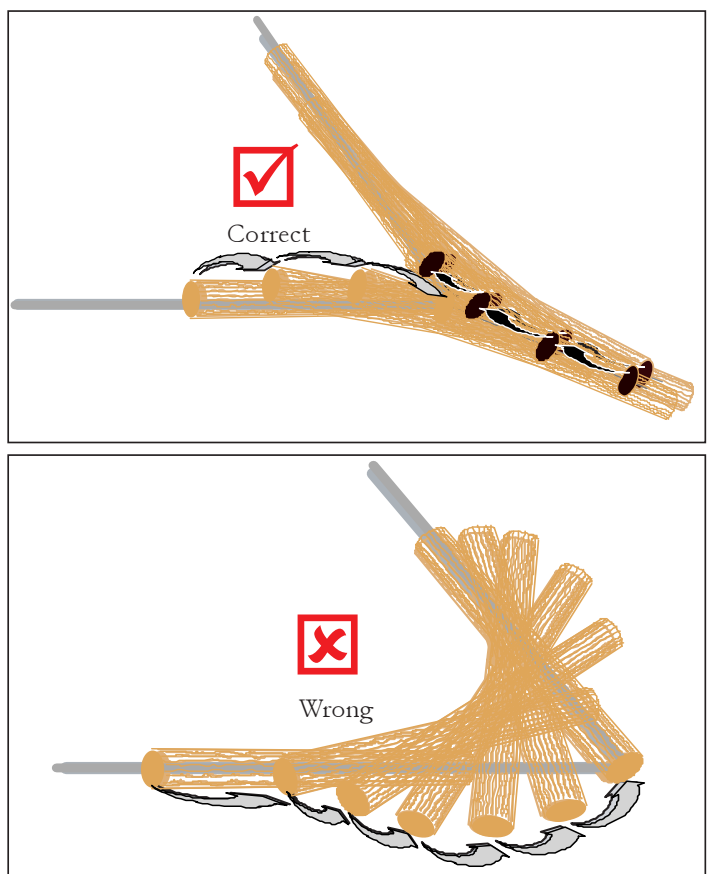


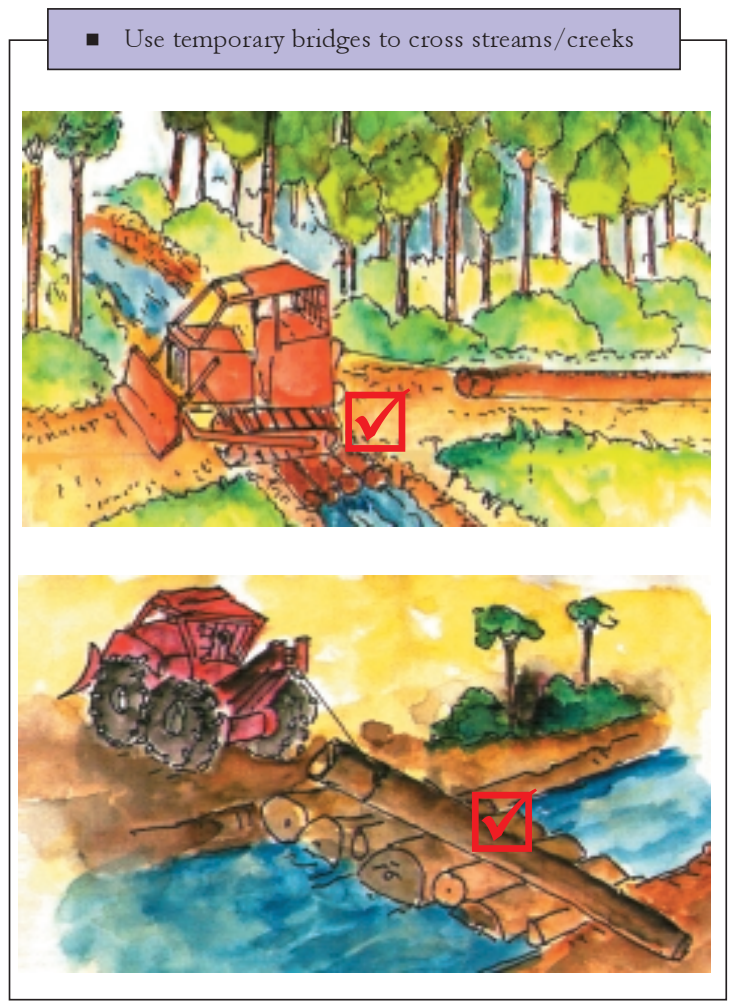




\section{Landing Operations}
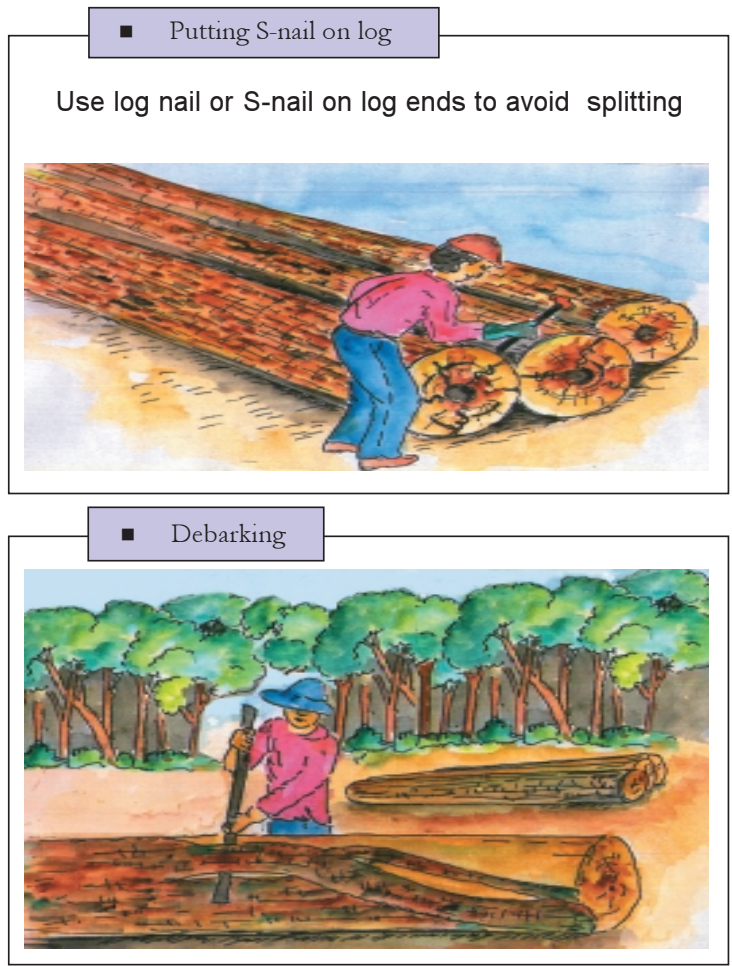


\section{Log storage}

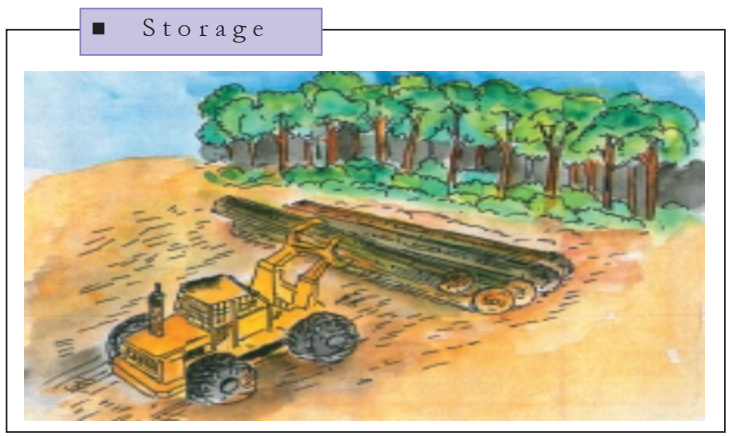

Loading

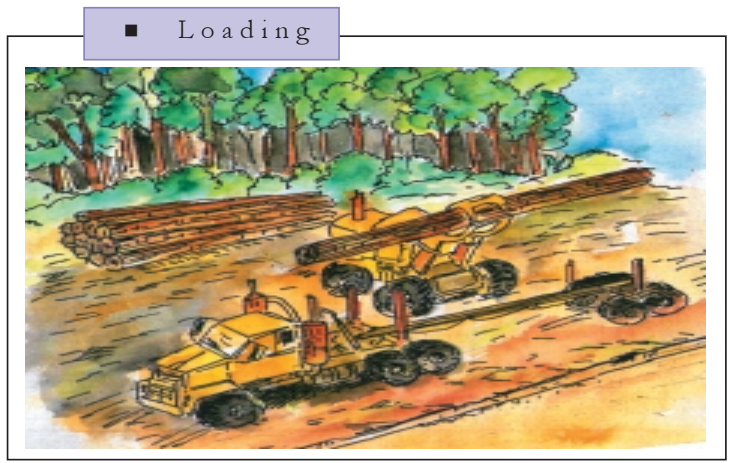




\section{MAINTENANCE, CAMP HYGIENE AND SAFETY}

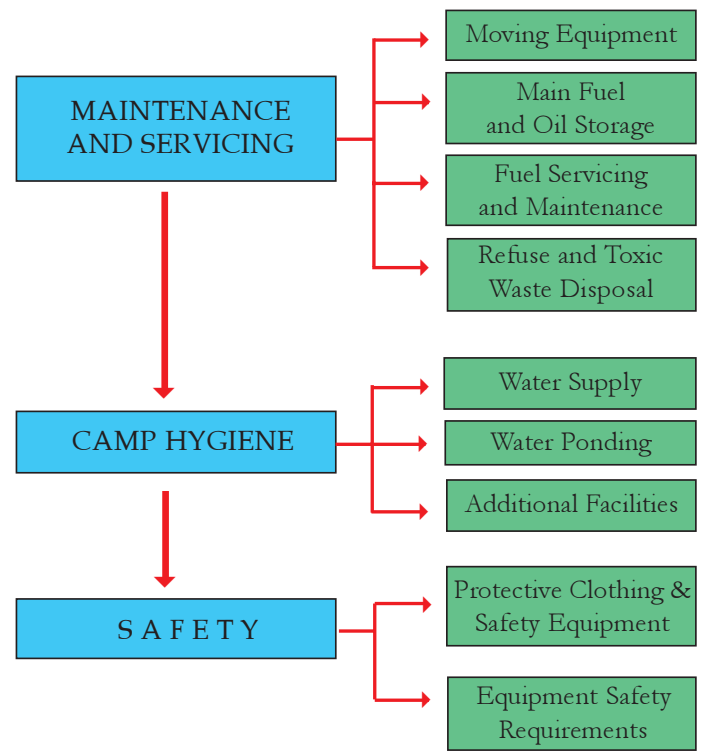




\section{MAINTENANCE AND SERVICING}

\section{Moving Equipment}

- All equipment is to be washed thoroughly before it is moved to a new operational area

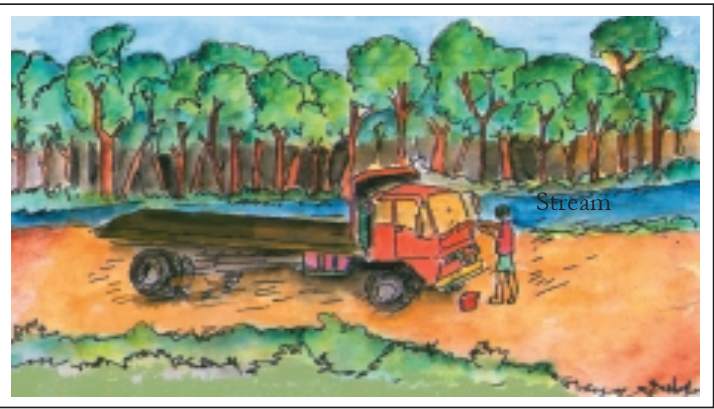




\section{Main Fuel and Oil Storage}

- Locate in a well-drained area and not less than $100 \mathrm{~m}$ from village areas and $50 \mathrm{~m}$ from watercourses

- When they must be located on the wharf, they should be:

* Located as far away from the sea as the fuel pumping lines permit

* Fitted with a proper, leak-proof bund, which in the event of a spill can be pumped to a disposal pit

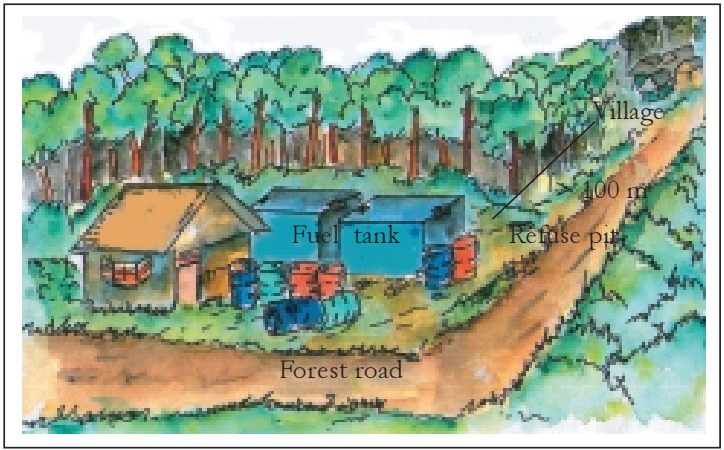




\section{Fuel Servicing \\ and Maintenance}

- Field fuel tank, refuelled points and maintenance areas are to be located:

* In well-drained areas such as on landings or road junctions

* Outside areas excluded from logging

- Sump oil is not to be dumped in the logging areas, but should be collected and removed to the main disposal facility

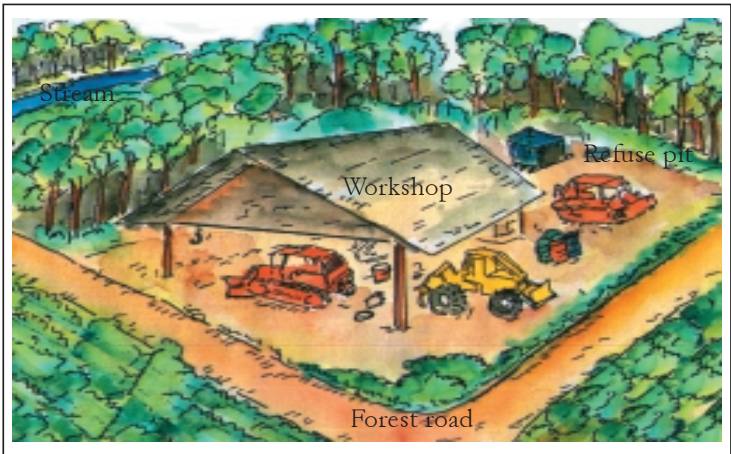




\section{Refuse and Toxic Waste Disposal}

- Refuse pits should be located at least $100 \mathrm{~m}$ from areas subject to inundation by water and at least $1 \mathrm{~m}$ above maximum ground water height

- The refuse pit location shoud be clearly indicated (including signs in all the local languages) and the pit securely fenced

- Toxic materials are to be collected in containers and sealed securely

- All solid waste should be placed in refuse pits and buried as soon as harvesting in the coupe has been completed

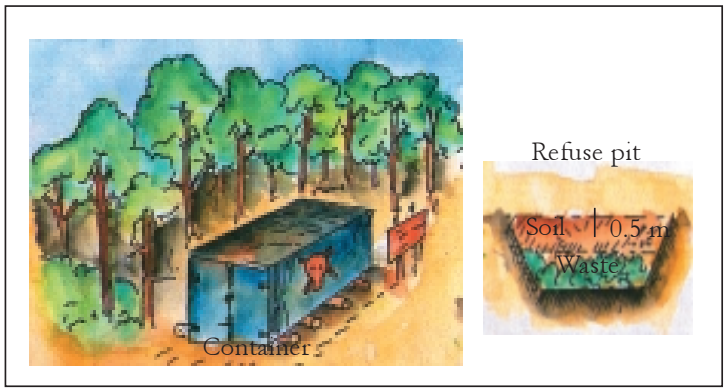



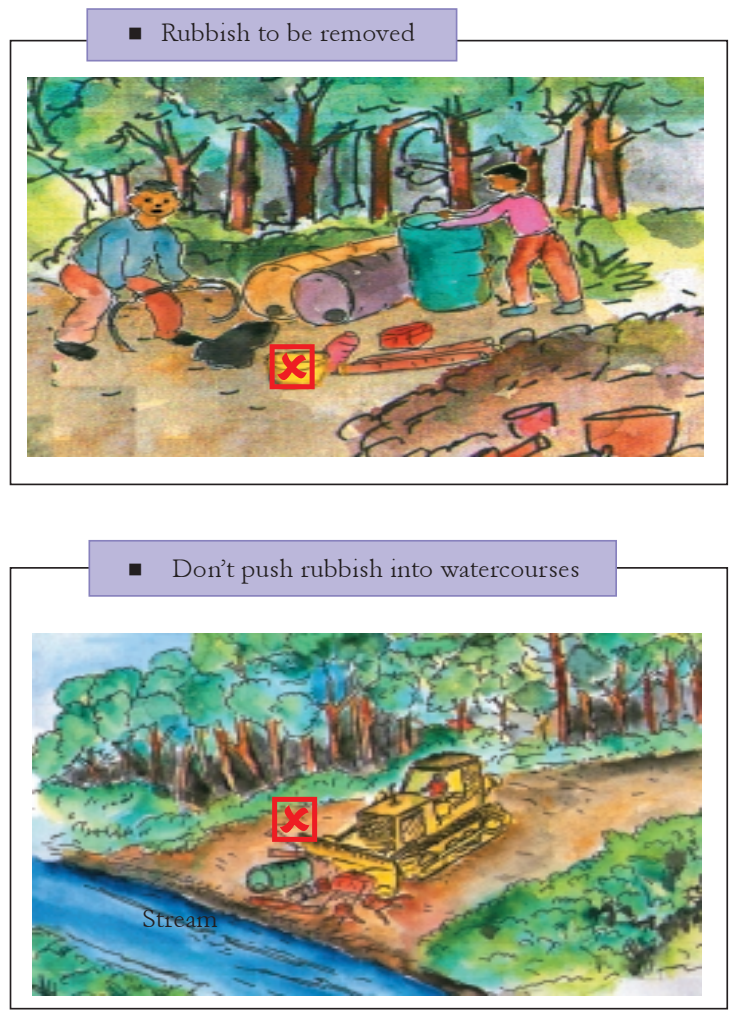


\section{CAMP HYGIENE}

\section{Water Supply}

- Camp should be supplied with clean water obtained from running streams, rainwater or wells

- Water storage tanks should be properly screened

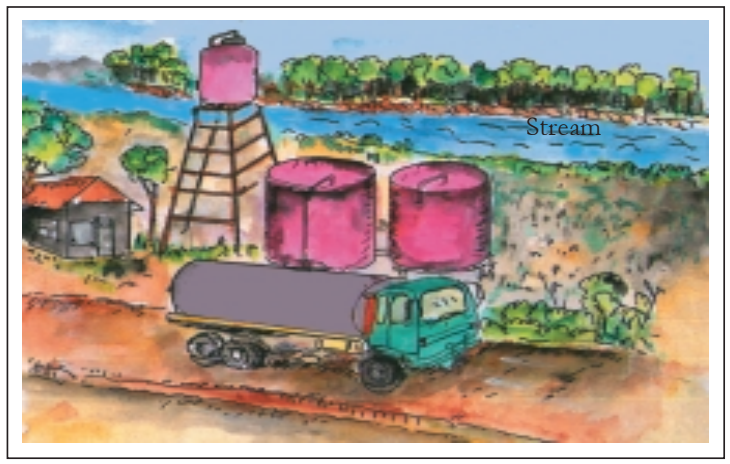




\section{Water Ponding}

- Camp areas should be well-drained, to avoid water impoundment that creates mosquito breeding areas

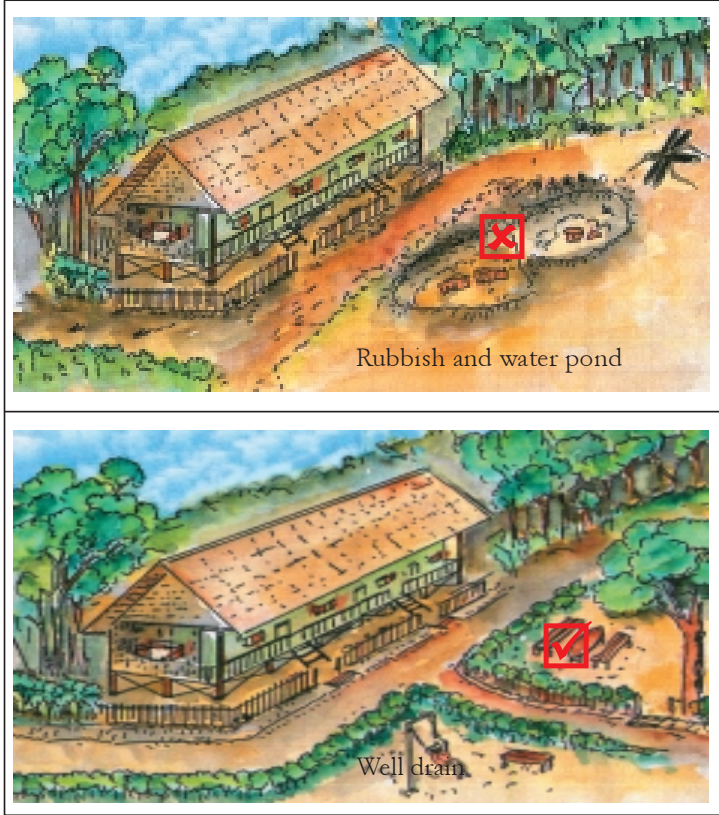




\section{Additional Facilities}

- Additional facilities in camps should include:

* Medical

* Educational

* Recreational, and

* Spiritual facilities

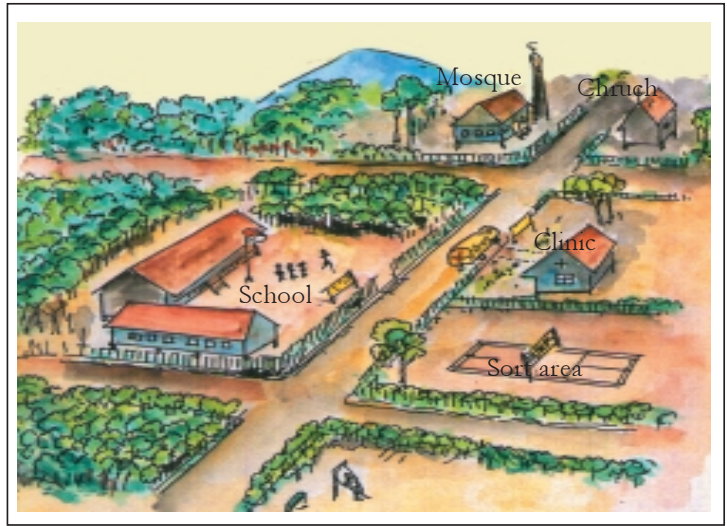




\section{SAFETY}

\section{Protective Clothing and Safety Equipment}

Guide to protective clothing

\begin{tabular}{|c|c|c|c|c|c|c|c|c|}
\hline $\begin{array}{l}\text { Logging } \\
\text { Crew }\end{array}$ & $\begin{array}{l}\text { Safety } \\
\text { Boots }\end{array}$ & $\begin{array}{c}\text { Safety } \\
\text { Trousers }\end{array}$ & $\begin{array}{l}\text { Close-fit } \\
\text { Clothing }\end{array}$ & $\begin{array}{c}\text { High } \\
\text { Visibility } \\
\text { Clothing }\end{array}$ & Gloves & $\begin{array}{l}\text { Safety } \\
\text { Helmet }\end{array}$ & Visor & $\begin{array}{l}\text { Ear } \\
\text { Muffs }\end{array}$ \\
\hline $\begin{array}{l}\text { Chainsaw } \\
\text { Operator }\end{array}$ & $\sqrt{ }$ & $\sqrt{ }$ & $\sqrt{ }$ & $\sqrt{ }$ & $\sqrt{ }$ & $\sqrt{ }$ & $\sqrt{ }$ & $\sqrt{ }$ \\
\hline $\begin{array}{l}\text { Tractor } \\
\text { Operator }\end{array}$ & $V$ & & $\sqrt{ }$ & $\sqrt{ }$ & $\sqrt{ }$ & $\sqrt{ }$ & & $\sqrt{ }$ \\
\hline $\begin{array}{l}\text { Loader } \\
\text { Operator }\end{array}$ & $\sqrt{ }$ & & $\sqrt{ }$ & $\sqrt{ }$ & $\sqrt{ }$ & $\sqrt{ }$ & & $\sqrt{ }$ \\
\hline Helper & $\sqrt{ }$ & & $\sqrt{ }$ & $\sqrt{ }$ & $\sqrt{ }$ & $\sqrt{ }$ & & $\sqrt{ }$ \\
\hline
\end{tabular}

\section{Equipment Safety \\ Requirement}

- Minimum safety standard

\section{Chainsaw:}

- Clearly marked positive on-off switch

- Safety throttle catch

- Chainsaw cutter and chain brake guard

- Exhaust system to direct fumes away from the operator

- Tool kit 


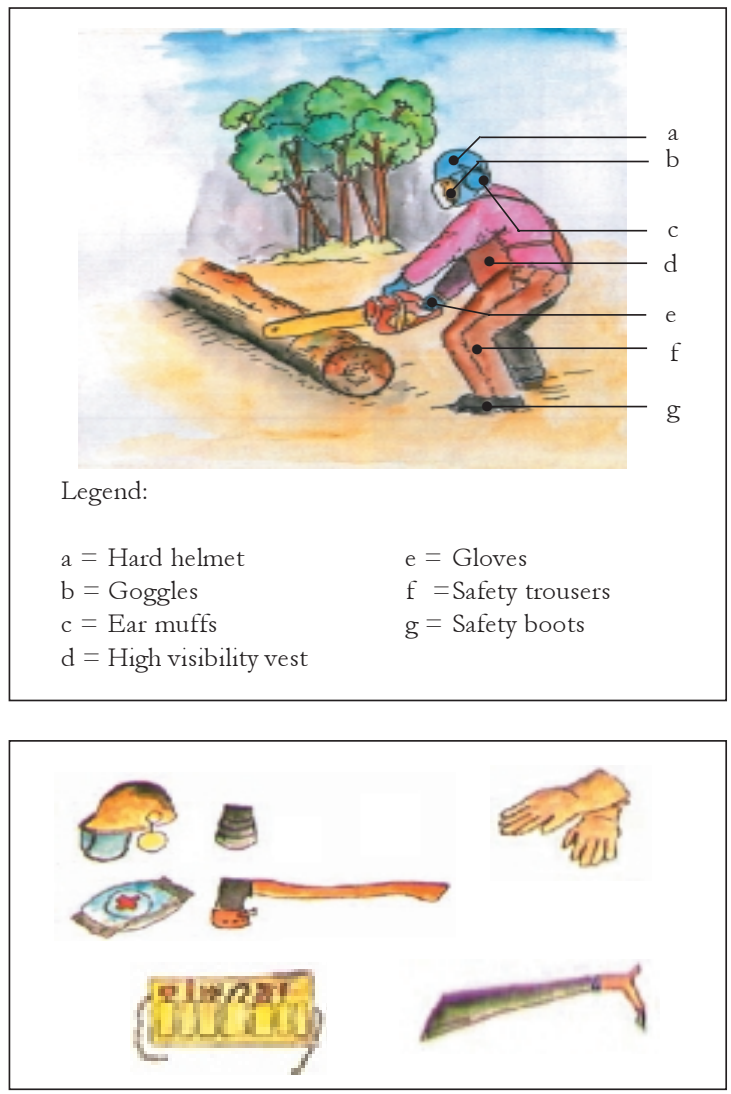


- Minimum safety standard

\section{Tractor:}

- Roll Over Protection Structure (ROPS)

- Falling Object Protection Structure (FOPS)

- Securely mounted seat and seat belt

- Rear of cab or frame fitted with protective wire mesh

- Reverse alarms

- Securely guarded pulleys, shafts, belts and fan blades

- Positive, non-returning engine stopping devices

- Suitable fire extinguisher firmly fixed and easily retrievable

- Exhaust system fitted with a spark arrestor

- First aid 


\section{POST-LOGGING ACTIVITIES}

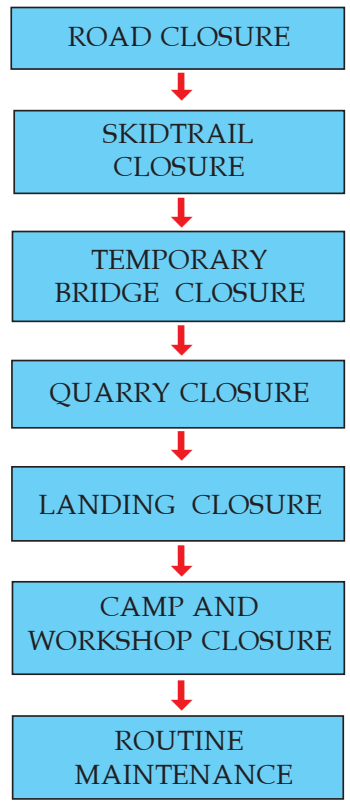




\section{ROAD CLOSURE}

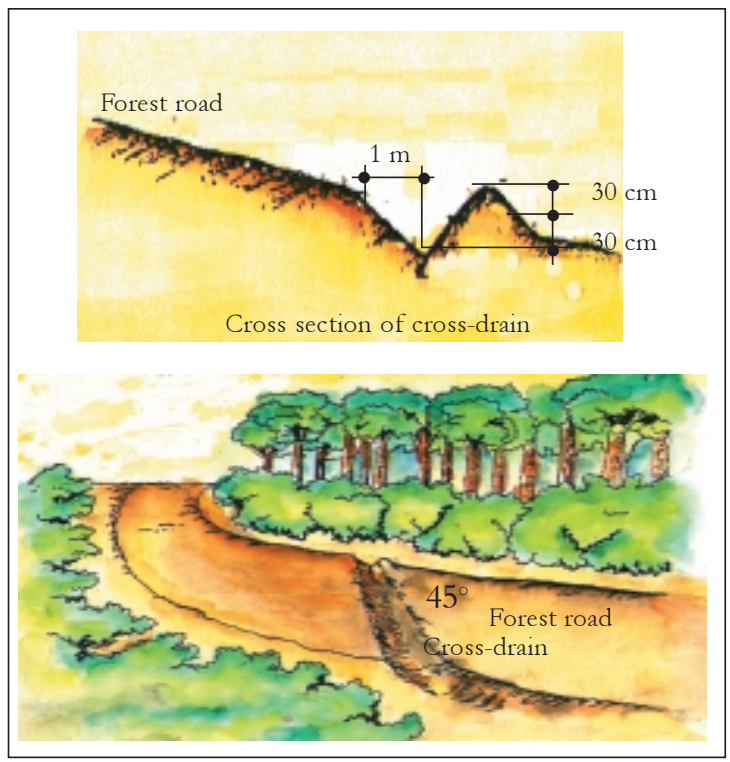




\section{SKIDTRAIL CLOSURE}

- Skidtrail cross-drain construction with interval of $20-30 \mathrm{~m}$

Cross-drains should be directed towards the undisturbed part of the forest
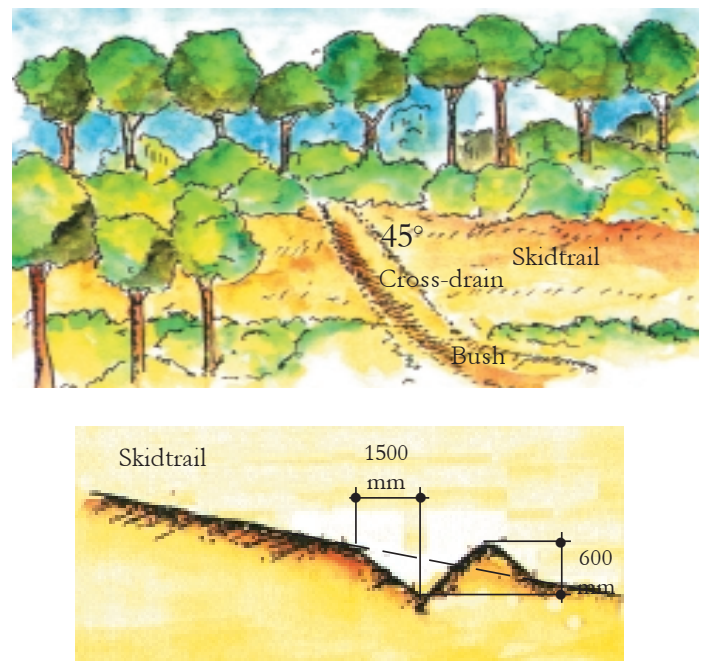

Cross section of skidtrail cross-drain 


\section{TEMPORARY BRIDGE CLOSURE}

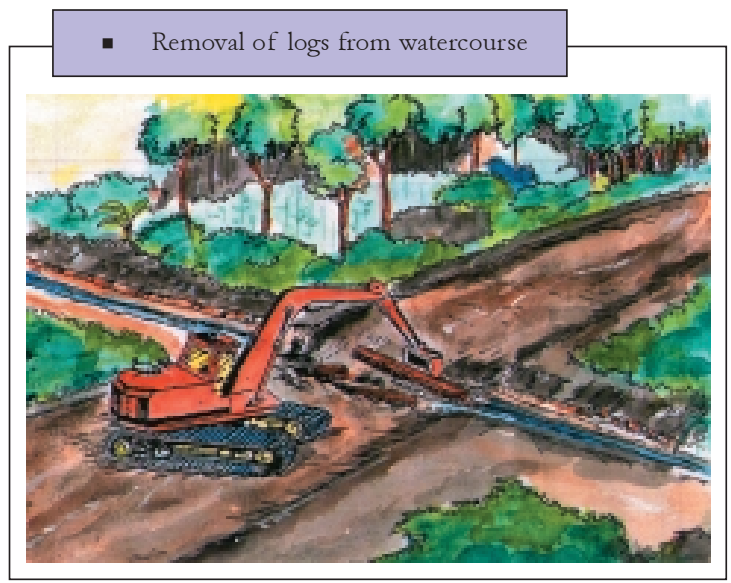



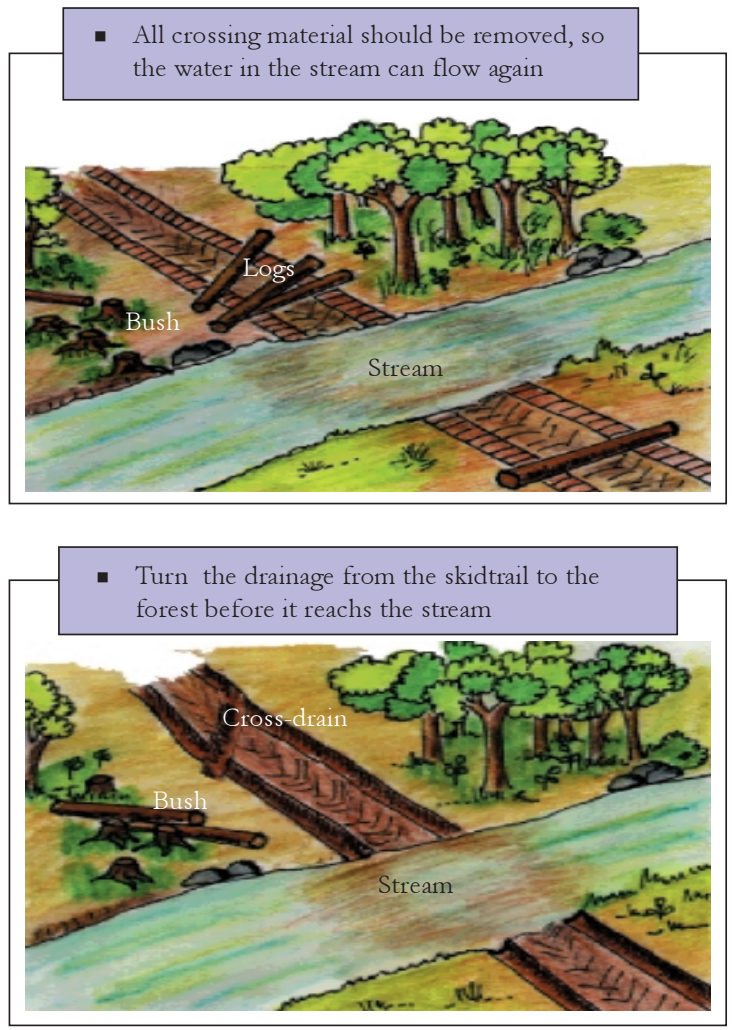


\section{QUARRY CLOSURE}
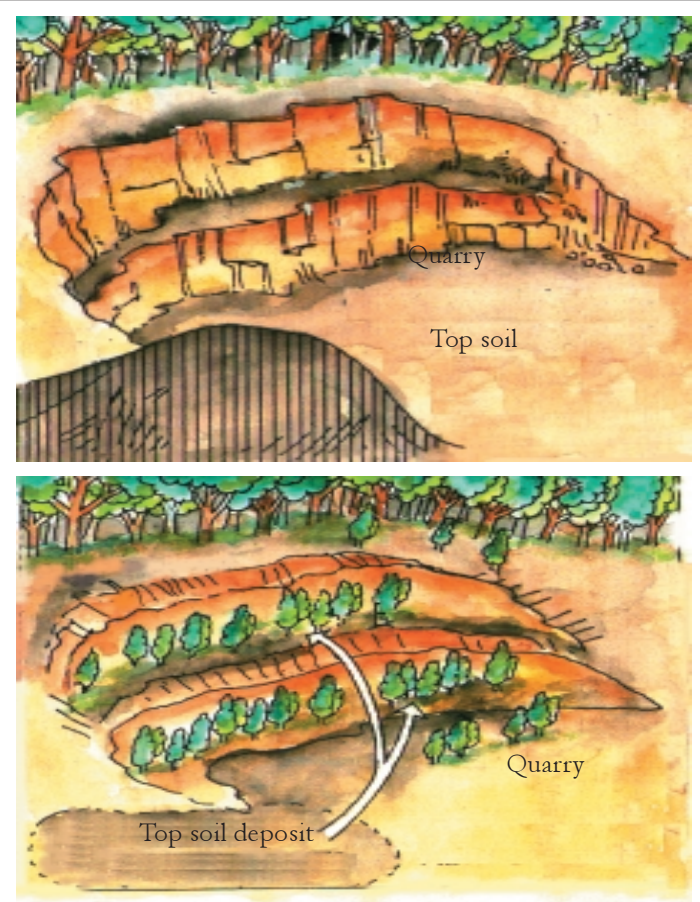


\section{LANDING CLOSURE}
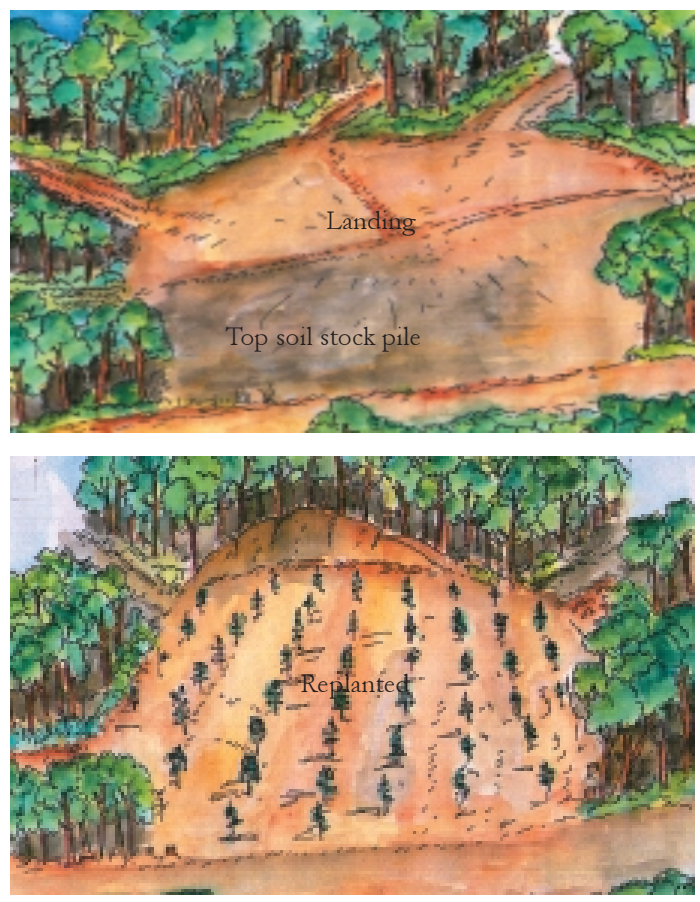


\section{CAMP AND WORKSHOP CLOSURE}

- Remove all refuse from camp and workshop areas

- All solid waste should be placed in a refuse pit and buried

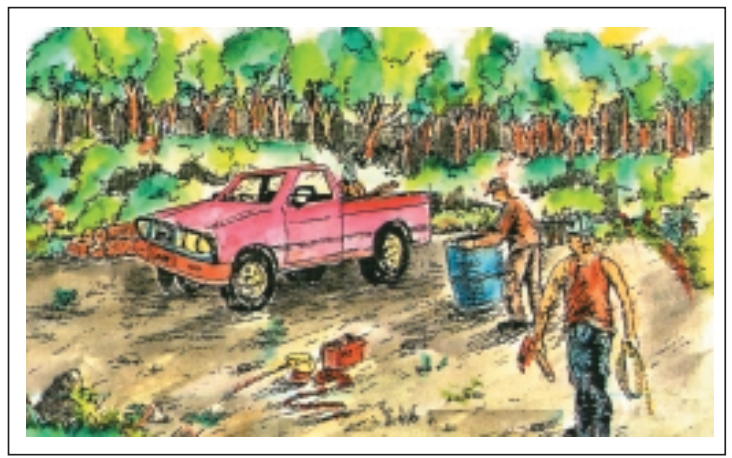




\section{ROAD MAINTENANCE}
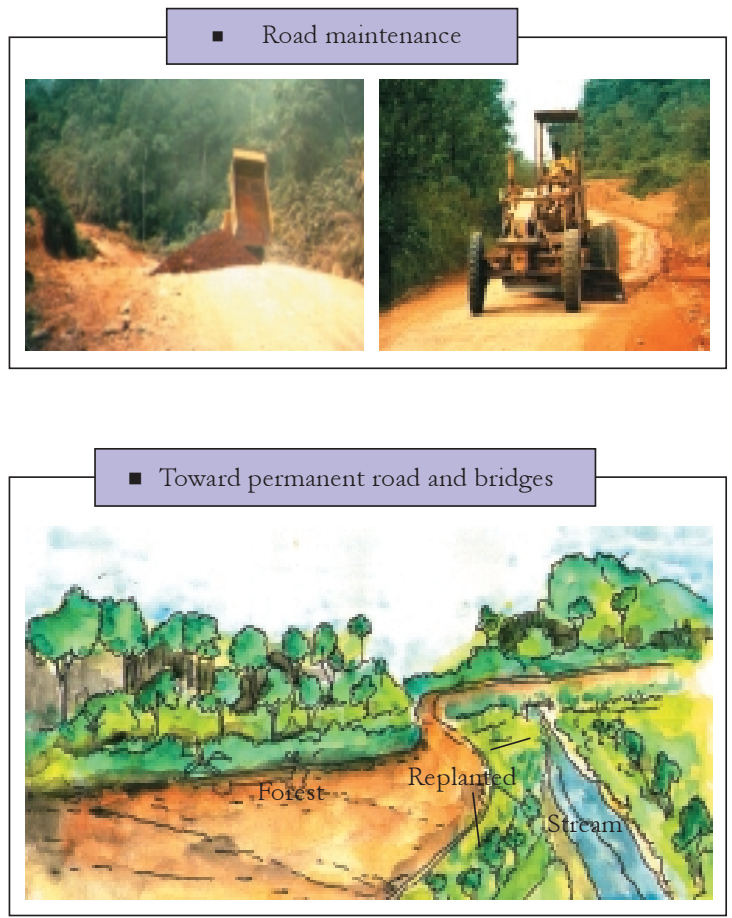
- Road drainage maintenance

- All roads should be graded on a regular basis

- Additional construction equipment such as rollers and watercarts may be used in conjuction with the grader to recompact the road surface

- Road maintenance may require periodic addition of surfacing materials

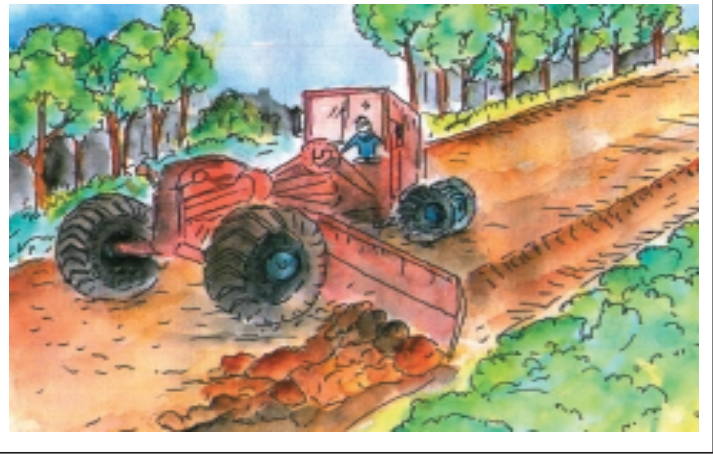


- Bridge maintenance

- Bridges should be inspected at least twice yearly, to confirm that:

* Waterway is clear of debris

* Substructure and superstructure are stable

* Wingwall protection is functional and that no erosion has occured

* Scour has not occurred adjacent to abutments

* Deck surface is in good condition

* Bridge approaches provide smooth access

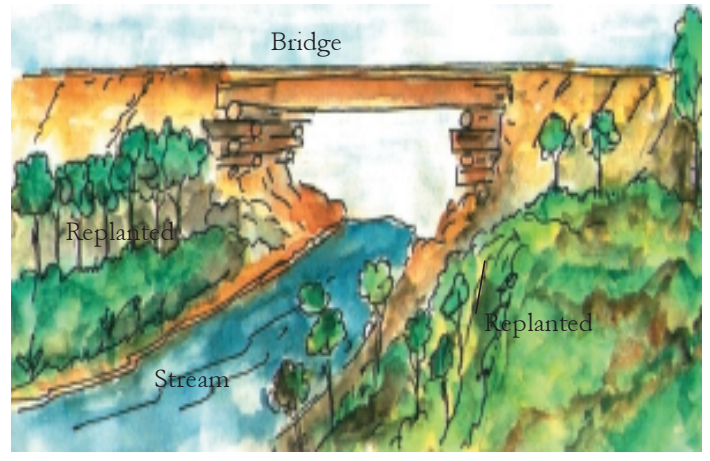




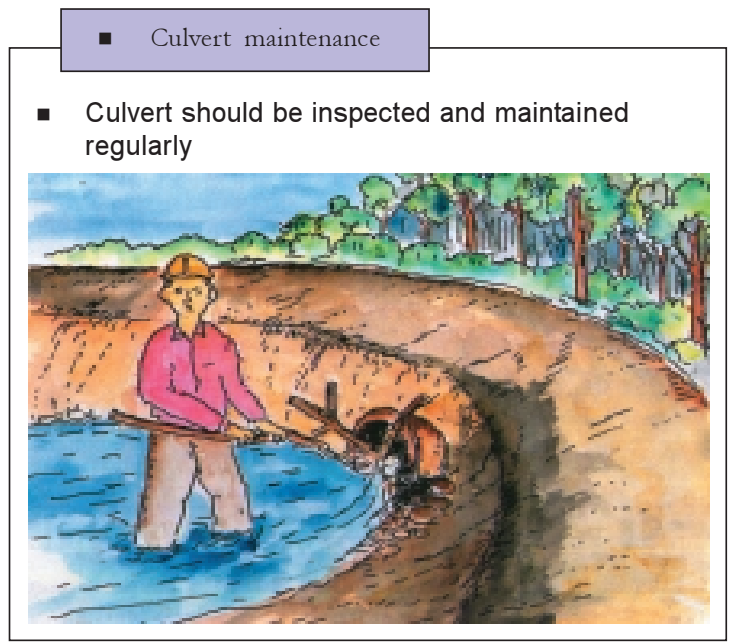




\section{CITED REFERENCES}

Departemen Kehutanan, RI. 1993. Pedoman dan Petunjuk Teknis Tebang Pilih Tanam Indonesia (TPTI) pada Hutan Alam Daratan. Departemen Kehutanan, Direktorat Jenderal Pengusahaan Hutan, Jakarta, Indonesia.

Elias. 1997. Pembukaan Wilayah Hutan. Fakultas Kehutanan IPB, Bogor, Indonesia.

FAO. undated. A Manual for Planning, Design and Construction of Forest Roads in Steep Terrain. FAO, Rome, Italy.

Ruslim, Y. 1998. Petunjuk Dasar dalam Timber Cruising dan Survei Topografi. SFMP Document No. 16(1998). Promotion of Sustainable Forest Management Systems (SFMP) in East Kalimanta.

TFF and APHI. 2001. Prosedur Survey Topografi Hutan. Tropical Forest Foundation bekerjasama dengan Asosiasi Pengusaha Hutan Indonesia, Indonesia 


\section{FURTHER READING}

Asia-Pacific Forest Commission. 1999. Code of Practice for Harvesting in Asia-Pacific. RAP Publication 1999/12, Bangkok, Thailand.

Departemen Kehutanan, RI dan APHI. 1997. Buku Petunjuk

Penggunaan Program "Forest Inventory-End Product Lingking

Programme" (FIEPLP). Modul Pelatihan di Wanariset Semboja, Kalimantan Timur, Indonesia.

Department of Forests, Vanuatu. 1997. Vanuatu Reduced Impact

Logging Guidelines. December, 1997. Vanuatu.

Department of Forestry and Wildlife, Kingdom of Cambodia. 1999.

The Cambodian Code of Practice for Forest Harvesting.

Cambodia.

Department of Foresty and Estate Crops, RI and NRM Program

Foresty Office. 2000. Principles and Practices for Forest Harvesting in Indonesia. Indonesia.

Elias. 1999. Buku Saku Reduced Impact Logging. PT. Penebar Swadaya, Jakarta, Indonesia.

Elias. 1999. Reduced Impact Timber Harvesting in the Indonesian Selective Cutting and Planting System. IPB Press, Bogor, Indonesia.

Elias. 1998. Reduced Impact Timber Harvesting in the Tropical Natural Forest in Indonesia. FAO, Rome, Italy.

FAO. 1998. Guidelines for Management of Tropical Forests. Rome, Italy.

Klasson,B. undated. Felling Guidelines for Reduced Impact Logging in Tropical Moist Forest. unpublished report.

Kusmaryono, B., J.R. Watulangkow dan H. Prayudi. 1997. Petunjuk Pengukuran Lapangan "Forest Inventory-End Product Linking Programme' (FIEPLP). Modul Pelatihan di Wanariset Semboja, Kalimantan Timur, Indonesia, Juni 1997.

Sist, P., Dykstra, D.P., Fimbel, R. 1998. Ground-Based ReducedImpact Logging Guidelines for Lowland and Hill Dipterocarp Forests in Indonesia. CIFOR, Occacional Paper, No. 15. 